\title{
Synthesis of Structural Elements of the Capsular Polysaccharide of Streptococcus pneumoniae Type 8
}

\author{
Franciscus A. W. Koeman, Johannis P. Kamerling*, and Johannes F. G. Vliegenthart \\ Bijvoet Center, Department of Bio-Organic Chemistry, Utrecht University, \\ P.O. Box 80.075 , NL-3508 TB Utrecht, The Netherlands
}

(Received in UK 18 March 1993)

\begin{abstract}
The synthesis is reported of propyl 4-O- $\alpha$-D-galactopyranosyl- $\beta$-D-glucopyranosiduronic acid (25), 4-O-[4$O$-( $\beta$-D-glucopyranosyluronic acid)- $\beta$-D-glucopyranosyl]-D-glucopyranose (34), and 4-O-(4-O- $\beta$-D-glucopyranosyl- $\alpha$-Dglucopyranosyl)-D-galactopyranose (38), each representing a structural element of the repeating unit of the capsular

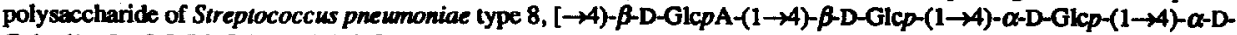
Galp- $(1 \rightarrow]_{\mathrm{n}}$ - 2,3-Di- $O$-benzyl-4,6-O-benzylidene- $\alpha$-D-galactopyranosyl trichloroacetimidate (12) was coupled to allyl 2- $O$-acetyl-3-O-benzyl-6- $O$-trityl- $\beta$-D-glucopyranoside $(7)$ in dichloromethane-ether, using trimethylsilyl trifluoromethanesulfonate as a promoter, in give disaccharide derivative 20 . Detritylation of $\mathbf{2 0}$, followed by oxidation and deprotection, afforded disaccharide propyl glycoside 25 . Coupling of 2,3,4,6-tetra-O-acetyl- $\alpha$-D-glucopyranosyl trichloroacetimidate (8) to ally1 2,3,6-tri-O-benzyl-4-O-(2,3,6-tri-O-benzyl- $\beta$-D-glucopyranosyl)- $\beta$-D-glucopyranoside (17) in dichloromethane, with trimethylsilyl trifluoromethanesulfonate as a promoter, resulted in trisaccharide derivative 26. Deacetylation of 26, followed by 6-O-tritylation, benzylation, detritylation, and oxidation gave a protected trisaccharide derivative (31), which, after deprotection, afforded 34. Coupling of ally1 2,3,6-tri- $O$-benzyl- $\beta$-D-galactopyranoside (10) to 4-O-(2,3-di-O-benzyl-4,6-O-benzylidene- $\beta$-D-glucopyranosyl)-2,3,6-tri-O-benzyl-D-glucopyranosyl trichloroacetimidate (19) in ether, with trimethylsilyl trifluoromethanesulfonate as a promoter, gave trisaccharide derivative 35. Deallylation of 35, followed by hydrogenolysis afforded 38.
\end{abstract}

\section{INTRODUCTION}

The current polysaccharide vaccine Pneumovax 23 against pneumococcal diseases such as pneumonia, otitis media, and meningitis, contains a mixture of the purified capsular polysaccharides of 23 serotypes ${ }^{1}$ of Streptococcus pneumoniae. This selection of 23 serotypes, of the 85 different serotypes known today, covers $90 \%$ of all pneumococcal infections. In view of the immunological problems ${ }^{2}$ related to this vaccine, attention is paid to the preparation of better alternatives based on oligosaccharide-conjugates. One of the constituents of the current vaccine is the capsular polysaccharide of serotype 8 , of which the structure has been characterised ${ }^{3}$ as:

$$
[\rightarrow 4)-\beta-\mathrm{D}-\mathrm{Glc} p \mathrm{~A}-(1 \rightarrow 4)-\beta-\mathrm{D}-\mathrm{Glc} p-(1 \rightarrow 4)-\alpha-\mathrm{D}-\mathrm{Glc} p-(1 \rightarrow 4)-\alpha-\mathrm{D}-\mathrm{Gal} p-(1 \rightarrow]_{\mathrm{n}}
$$


Here we report on the synthesis of three structural elements of the capsular polysaccharide of serotype 8, namely, propyl 4-O- $\alpha$-D-galactopyranosyl- $\beta$-D-glucopyranosiduronic acid (25), 4-O-[4-O-( $\beta$-D-glucopyranosyluronic acid)- $\beta$-D-glucopyranosyl]-D-glucopyranose (34), and 4-O-(4-O- $\beta$-D-glucopyranosyl- $\alpha$-D-glucopyranosyl)-D-galactopyranose (38). During the preparation of this manuscript the synthesis of two protected fragments of the capsular polysaccharide 4 was reported.

\section{RESULTS AND DISCUSSION}

The tetrasaccharide repeating unit of the capsular polysaccharide of $S$. pneumoniae serotype 8 can be divided into a glucuronic acid, a galactose, and a cellobiose element. For the synthesis of the aimed oligosaccharides 25,34 , and 38 , which form overlapping fragments of the tetrasaccharide repeating unit, a series of suitably protected synthons was prepared. For the glucuronic acid element allyl 2-O-acetyl-3-O-benzyl-6- $O$ trityl- $\beta$-D-glucopyranoside (7) and 2,3,4,6-tetra- $O$-acetyl- $\alpha$-D-glucopyranosyl trichloroacetimidate (8) were synthesised, for the galactose element allyl 2,3,6-tri- $O$-benzyl- $\beta$-D-galactopyranoside $(10)$ and 2,3-di- $O$-benzyl-4,6-O-benzylidene- $\alpha$-D-galactopyranosyl trichloroacetimidate (12), and for the cellobiose part allyl 2,3,6tri- $O$-benzyl-4-O-(2,3,6-tri- $O$-benzyl- $\beta$-D-glucopyranosyl)- $\beta$-D-glucopyranoside (17) and 4-O-(2,3-di- $O$-benzyl-4,6-O-benzylidene- $\beta$-D-glucopyranosyl)-2,3,6-tri- $O$-benzyl-D-glucopyranosyl trichloroacetimidate (19).

Complete deacetylation of allyl 2,4,6-tri- $O$-acetyl-3-O-benzyl- $\beta$-D-glucopyranoside ${ }^{5}(2)$ at strong alkaline

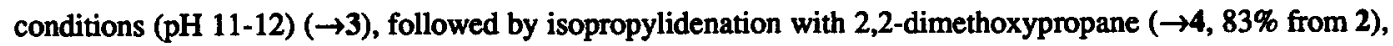
acetylation $(\rightarrow 5)$, and de-isopropylidenation gave $6(84 \%$ from 4$)$. Then 6 was tritylated with trityl chloride in pyridine to afford 'glucuronic acid acceptor' 7 (78\%). 'Glucuronic acid donor' 8 was synthesised by imidation of 2,3,4,6-tetra- $O$-acetyl-D-glucopyranose 6 using trichloroacetonitrile and 1,8-diazabicyclo[5.4.0]undec-7-ene 7 as a base in dichloromethane in a yield of $76 \%$.

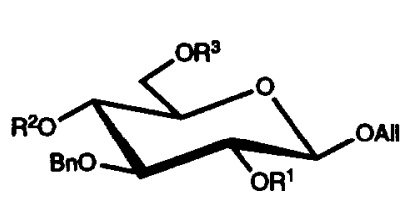
$2 \mathbf{R}^{1}=\mathbf{R}^{2}=\mathbf{R}^{3}=\mathrm{AC}$
$3 R^{1}=R^{2}=R^{3}=H$
$4 \mathrm{R}^{1}=\mathrm{H} ; \mathrm{R}^{2}, \mathrm{R}^{3}=\mathrm{C}\left(\mathrm{CH}_{3}\right)_{2}$
$5 \mathbf{R}^{1}=A c ; R^{2}, R^{3}=C\left(C_{3}\right)_{2}$
$6 R^{1}=A c ; R^{2}=R^{3}=H$
$7 \mathbf{R}^{1}=\mathrm{Ac} ; \mathrm{R}^{2}=\mathrm{H} ; \mathbf{R}^{3}=\mathrm{Tr}$

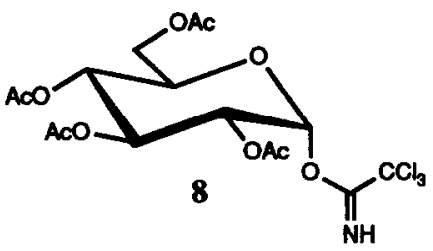

Both galactose synthons 10 and 12 were prepared from allyl 2,3-di- $O$-benzyl-4,6- $O$-benzylidene- $\beta$-Dgalactopyranoside ${ }^{8}(9)$, using the following sequence of reactions. Regioselective reductive ring opening of 9 with sodium cyanoborohydride and hydrogen chloride 9 afforded 'galactose acceptor' 10 (60\%). The 'galactose donor' 12 was prepared by deallylation of 9 via isomerisation using potassium tert-butoxide and depropenylation with mercuric oxide and mercuric chloride ${ }^{10}(\rightarrow 11,74 \%)$, and subsequent imidation using trichloroacetonitrile and 1,8-diazabicyclo[5.4.0]undec-7-ene $(\rightarrow 12,96 \%)$. 


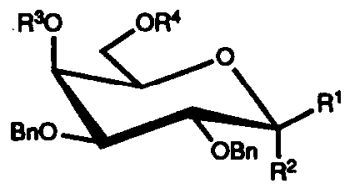

$9 R^{1}=$ OAll; $R^{2}=H ; R^{3}, R^{4}=C H P h$

$10 R^{1}=$ OAll; $R^{2}=R^{3}=H ; R^{4}=B n$

$11 R^{1}, R^{2}=H, O H ; R^{3}, R^{4}=C H P h$

$12 R^{1}=H ; R^{2}=\mathrm{OCNHCCl}_{3} ; R^{4}, \mathrm{R}^{5}=\mathrm{CHPh}$

For the synthesis of the cellobiose synthons 17 and 19, the same approach was followed as for the galactose synthons. Thus, allyl 4- $O$-(2,3,4,6-tetra- $O$-acetyl- $\beta$-D-glucopyranosyl)-2,3,6-tri- $O$-acetyl- $\beta$-D-glucopyranoside ${ }^{11}$ (13) was deacetylated ( $\rightarrow 14$, quantitative), 4,6-O-benzylidenated with benzaldehyde dimethyl acetal in $N, N$-dimethylformamide ( $\rightarrow 15$ ), and benzylated to give 16 (77\% from 14). Regioselective reductive ring opening of 16 with sodium cyanoborohydride and hydrogen chloride afforded 'cellobiose acceptor' 17 (78\%). Deallylation of $16(\rightarrow 18,93 \%)$, and subsequent imidation, gave 'cellobiose donor' 19 (79\%).

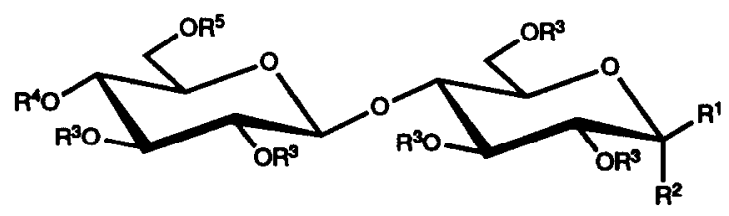

$13 \mathrm{R}^{1}=$ OAll; $\mathrm{R}^{2}=\mathrm{H} ; \mathrm{R}^{3}=\mathrm{R}^{4}=\mathrm{R}^{5}=\mathrm{Ac}$

$14 R^{1}=$ OAll; $R^{2}=R^{3}=R^{4}=R^{5}=H$

$15 R^{1}=$ OAll; $R^{2}=R^{3}=H ; R^{4}, R^{5}=$ CHPh

$16 R^{1}=$ OAll; $R^{2}=H ; R^{3}=B n ; R^{4}, R^{5}=C H P h$

$17 R^{1}=$ OAll; $R^{2}=R^{4}=H ; R^{3}=R^{5}=B n$

$18 \mathrm{R}^{1}, \mathrm{R}^{2}=\mathrm{H}, \mathrm{OH} ; \mathrm{R}^{3}=\mathrm{Bn} ; \mathrm{R}^{4}, \mathrm{R}^{5}=\mathrm{CHPh}$

$19 \mathrm{R}^{1}, \mathrm{R}^{2}=\mathrm{H}, \mathrm{OCNHCC} ; \mathrm{R}^{3}=\mathrm{Bn} ; \mathrm{R}^{4}, \mathrm{R}^{5}=\mathrm{CHPh}$

Coupling of 'galactose donor' 12 to 'glucuronic acid acceptor' 7 in dichloromethane-ether at $-70^{\circ}$, using trimethylsilyl trifluoromethanesulfonate as a promoter, gave allyl 2-O-acetyl-3-O-benzyl-4-O-(2,3-di-O-benzyl4,6- $O$-benzylidene- $\alpha$-D-galactopyranosyl)-6- $O$-trityl- $\beta$-D-glucopyranoside $(20,60 \%)$. After detritylation of 20 with perchloric acid ${ }^{12}(\rightarrow 21,76 \%)$, and oxidation with the Jones-reagent ${ }^{13}(\rightarrow 22)$, a methylation was carried out with diazomethane for separation purposes $(\rightarrow 23,70 \%)$. Because complete deacetylation of 23 with lithium hydroxide in water failed, a two-step saponification was used. To this end 23 was treated with sodium methoxide in methanol, to give a deacetylated product, and then with lithium hydroxide in water-acetone to remove the methyl ester group ( $\rightarrow 24,90 \%$ ). Finally, hydrogenolysis of 24 , leading to the removal of the benzyl groups and the benzylidene group, as well as to the conversion of the allyl group into a propyl group, afforded propyl 4- $O$ - $\alpha$-D-galactopyranosyl- $\beta$-D-glucopyranosiduronic acid $(25,73 \%)$. 


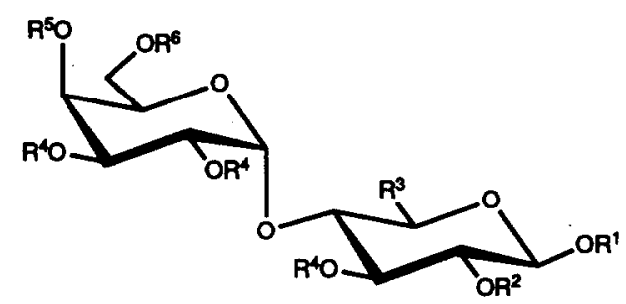

$20 \mathbf{R}^{1}=$ All; $\mathbf{R}^{2}=\mathrm{Ac} ; \mathbf{R}^{3}=\mathrm{CH}_{2} \mathrm{OTr}, \mathbf{R}^{4}=\mathrm{Bn} ; \mathbf{R}^{5}, \mathbf{R}^{6}=\mathrm{CHPh}$

$21 \mathbf{R}^{1}=$ All; $\mathbf{R}^{2}=\mathrm{Ac} ; \mathbf{R}^{3}=\mathrm{CH}_{2} \mathrm{OH} ; \mathbf{R}^{4}=\mathrm{Bn} ; \mathbf{R}^{5}, \mathbf{R}^{6}=\mathrm{CHPh}$

$22 \mathbf{R}^{1}=\mathrm{All} ; \mathbf{R}^{2}=\mathrm{Ac} ; \mathbf{R}^{3}=\mathrm{COOH} ; \mathbf{R}^{4}=\mathrm{Bn} ; \mathbf{R}^{5}, \mathbf{R}^{6}=\mathrm{CHPh}$

$23 \mathbf{R}^{1}=$ All; $R^{2}=A c ; R^{3}=$ COOMe; $R^{4}=B n ; R^{5}, R^{6}=C H P h$

$24 \mathrm{R}^{1}=\mathrm{All} ; \mathrm{R}^{2}=\mathrm{H} ; \mathrm{R}^{3}=\mathrm{COOH} ; \mathrm{R}^{4}=\mathrm{Bn} ; \mathrm{R}^{5}, \mathrm{R}^{6}=\mathrm{CHPh}$

$25 R^{1}=$ Propyl; $R^{2}=R^{4}=R^{5}=R^{6}=H ; R^{3}=C O O H$

Coupling of the 'glucuronic acid donor' 8 to 'cellobiose acceptor' 17 in dichloromethane at $-30^{\circ}$, with trimethylsilyl trifluoromethanesulfonate as a promoter, gave allyl 4-O-[4-O-(2,3,4,6-tetra-O-acetyl- $\beta$-D-glucopyranosyl)-2,3,6-tri- $O$-benzyl- $\beta$-D-glucopyranosyl]-2,3,6-tri- $O$-benzyl- $\beta$-D-glucopyranoside (26, 74\%). Deacetylation of $26(\rightarrow 27)$, followed by tritylation $(\rightarrow 28)$, benzylation $(\rightarrow 29,20 \%$ from 26$)$, and detritylation with perchloric acid ${ }^{12}$ gave $30(80 \%)$. Then oxidation with the Jones-reagent ${ }^{13}(\rightarrow 31,75 \%)$, followed by deallylation ${ }^{7}$ with palladium (II) chloride and sodium acetate in acetic acid $(\rightarrow 33,51 \%)$, and debenzylation yielded 4-O-[4-O-( $\beta$-D-glucopyranosyluronic acid)- $\beta$-D-glucopyranosyl]-D-glucopyranose $(34,90 \%)$. It has to be noted that during the deallylation step, 31 is partially converted into 32, having a saturated allyl (propyl) group.

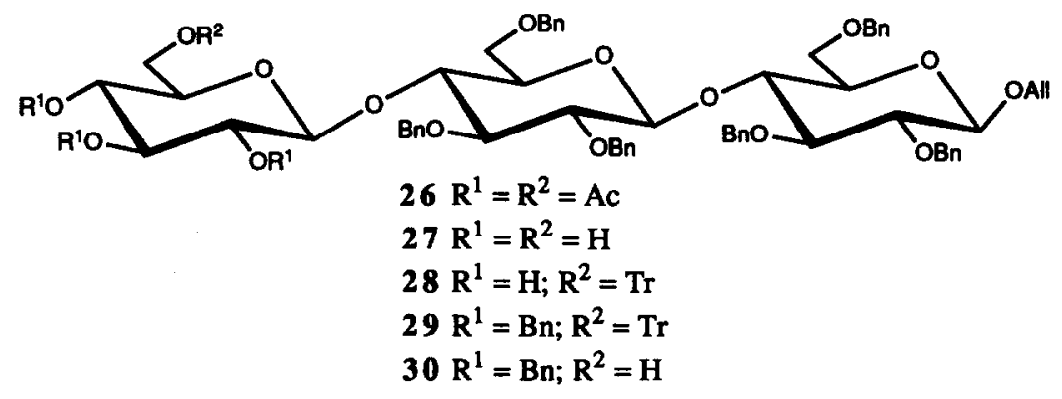




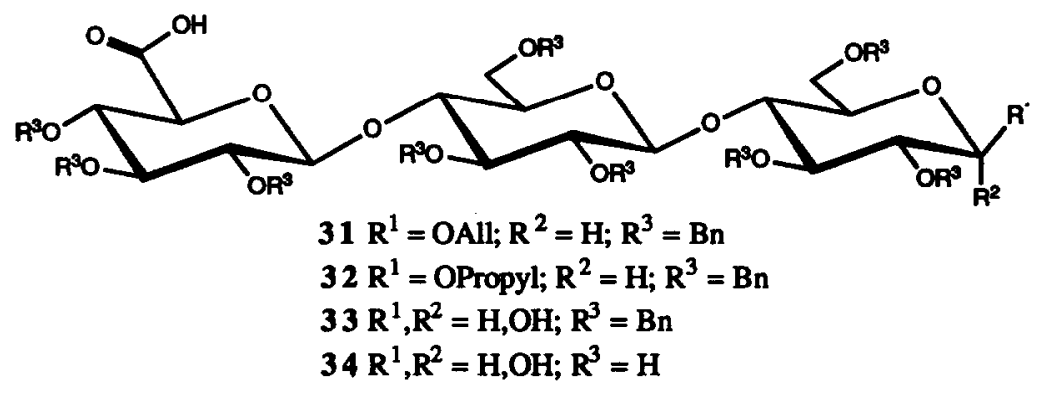

Coupling of 'cellobiose donor' 19 and 'galactose acceptor' 10 in ether at $-70^{\circ}$, using trimethylsilyl trifluoromethanesulfonate as a promoter, gave allyl 4-O-[4-O-(2,3-di-O-benzyl-4,6- $O$-benzylidene- $\beta$-D-glucopyranosyl)-2,3,6-tri- $O$-benzyl- $\alpha$-D-glucopyranosyl]-2,3,6-tri- $O$-benzyl- $\beta$-D-galactopyranoside $(35,55 \%)$, and its $\beta$-coupling product $(36,15 \%)$, which could be separated by column chromatography. Deallylation of 35 with potassium tert-butoxide, followed by mercuric oxide and mercuric chloride ${ }^{10}(\rightarrow 37,71 \%)$ and hydrogenation yielded 4-O-(4-O- $\beta$-D-glucopyranosyl- $\alpha$-D-glucopyranosyl)-D-galactopyranose $(38,80 \%)$. In a similar way also the $\beta$-coupling product was deallylated $(\rightarrow 39,62 \%)$ and debenzylated/debenzylidenated $(\rightarrow 40,77 \%)$.

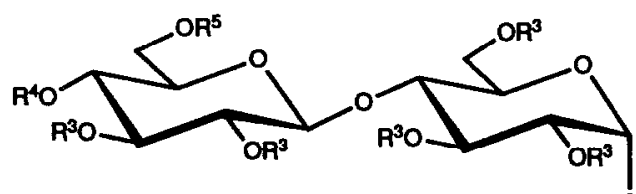

$35 \mathrm{R}^{1}=$ OAll; $\mathrm{R}^{2}=\mathrm{H} ; \mathrm{R}^{3}=\mathrm{Bn} ; \mathbf{R}^{4}, \mathrm{R}^{5}=\mathrm{CHPh}$

$37 \mathrm{R}^{1}, \mathrm{R}^{2}=\mathrm{H}, \mathrm{OH} ; \mathrm{R}^{3}=\mathrm{Bn} ; \mathrm{R}^{4}, \mathrm{R}^{5}=\mathrm{CHPh}$ $38 R^{1}, R^{2}=H, O H ; R^{3}=R^{4}=R^{5}=H$
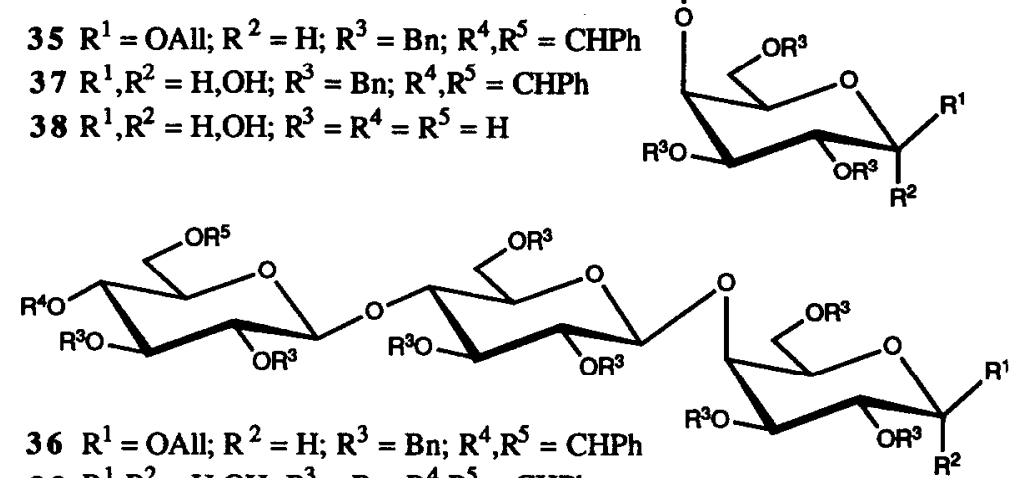

$39 R^{1}, R^{2}=H, O H ; R^{3}=B n ; R^{4}, R^{5}=C H P h$

$40 R^{1}, R^{2}=H, O H ; R^{3}=R^{4}=R^{5}=H$ 


\section{EXPERIMENTAL}

General methods.- ${ }^{1} \mathrm{H}-\mathrm{NMR}$ spectra were recorded at $300 \mathrm{MHz}$ with a Bruker AC 300 , at $360 \mathrm{MHz}$ with a Bruker HX 360, and at $500 \mathrm{MHz}$ with a Bruker AM 500 apparatus at 250. Two-dimensional doublequantum filtered ${ }^{1} \mathrm{H}_{-1}{ }^{1} \mathrm{H}$ correlation spectra (2D DQF ${ }^{1} \mathrm{H}-{ }^{1} \mathrm{H}$ COSY) were recorded in the phase-sensitive mode ${ }^{14}$, and two-dimensional homonuclear Hartmann-Hahn spectra (2D HOHAHA) with a $120 \mathrm{~ms} \mathrm{MLEV-17}$ mixing sequence ${ }^{15}$. ${ }^{13} \mathrm{C}-\mathrm{NMR}$ spectra (APT, $50 \mathrm{MHz}$ ) were recorded at $25^{\circ}$ with a Bruker WP 200 spectrometer. Chemical shifts $(\delta)$ are given in ppm relative to the signal for internal $\mathrm{Me}_{4} \mathrm{Si}\left(\mathrm{CDCl}_{3}\right)$ or internal sodium 4,4-dimethyl-4-silapentane-1-sulfonate $\left(\mathrm{D}_{2} \mathrm{O}\right.$; indirectly to internal acetone, $\left.\delta 2.225\right)$ for ${ }^{1} \mathrm{H}$, and to the signal for internal $\mathrm{Me}_{4} \mathrm{Si}\left(\mathrm{CDCl}_{3}\right.$; indirectly to $\left.\mathrm{CDCl}_{3}, \delta 76.9\right)$ or external $\mathrm{Me}_{4} \mathrm{Si}\left(\mathrm{D}_{2} \mathrm{O}\right.$; indirectly to internal acetone, $\delta$ 31.55) for ${ }^{13} \mathrm{C}$.

Column chromatography was performed on Kieselgel 60 (Merck, $<230$ mesh) and fractions were monitored by TLC on Kieselgel $60 \mathrm{~F}_{254}$ (Merck). Detection was effected by examination under UV light and by charring with aq $50 \%$ sulfuric acid. Optical rotations were measured at $20^{\circ}$ with a Perkin-Elmer 241 polarimeter, using a 10-cm 1-mL cell. In working-up procedures, washings were carried out three times with appropriate quantities of water or aq $10 \%$ sodium hydrogencarbonate unless indicated otherwise. The Jones reagent, used in oxidation reactions, consisted of a mixture of chromium (VI) oxide (76.7 g), conc. $\mathrm{H}_{2} \mathrm{SO}_{4}(28.5 \mathrm{~mL}$ ), and water $(111.5 \mathrm{~mL})$. Evaporations were conducted under reduced pressure at $400^{\circ}$. All solvents were distilled from appropriate drying agents.

Allyl 3-O-benzyl-4,6-O-isopropylidene- $\beta$-D-glucopyranoside (4). - To a solution of allyl 2,4,6-tri-Oacetyl-3-O-benzyl- $\beta$-D-glucopyranoside $5(2,8.5 \mathrm{~g}, 19.5 \mathrm{mmol})$ in $\mathrm{CH}_{2} \mathrm{Cl}_{2}(25 \mathrm{~mL})$ and $\mathrm{MeOH}(60 \mathrm{~mL})$ was added sodium methoxide ( $\mathrm{pH}$ 11-12). After stirring overnight the solution was neutralised with Dowex-50 $\left(\mathrm{H}^{+}\right)$ resin, filtered, and concentrated $(\rightarrow 3$, quantitative). To a solution of the residue in acetone $(30 \mathrm{~mL})$ were added 2,2-dimethoxypropane (70 $\mathrm{mL}$ ) and a catalytic amount of $p$-toluenesulfonic acid, and the mixture was stirred for $3 \mathrm{~h}$ to give a complete reaction (TLC 9:1 $\mathrm{CH}_{2} \mathrm{Cl}_{2}$-acetone; $4 R_{\mathrm{F}}$ 0.37). The mixture was neutralised with solid sodium hydrogencarbonate, filtered, and concentrated. A solution of the residue in $\mathrm{CH}_{2} \mathrm{Cl}_{2}(50 \mathrm{~mL}$ ) was washed with water, aq $10 \%$ sodium hydrogencarbonate, and water, dried $\left(\mathrm{MgSO}_{4}\right)$, filtered, and concentrated. Column chromatography (95:5 $\mathrm{CH}_{2} \mathrm{Cl}_{2}$-acetone) of the residue gave 4 , isolated as a white solid $(5.7 \mathrm{~g}, 83 \%)$, $[\alpha]_{\mathrm{D}}-17^{\circ}\left(c 1, \mathrm{CH}_{2} \mathrm{Cl}_{2}\right)$. NMR data $\left(\mathrm{CDCl}_{3}\right):{ }^{13} \mathrm{C}, \delta 133.5\left(\mathrm{OCH}_{2} \mathrm{CH}=\mathrm{CH}_{2}\right), 128.3-127.6(\mathrm{Ph}), 118.1$ $\left(\mathrm{OCH}_{2} \mathrm{CH}=\mathrm{CH}_{2}\right), 102.2(\mathrm{C}-1), 99.3\left(\mathrm{C}\left(\mathrm{CH}_{3}\right)_{2}\right), 80.7,74.0(2 \mathrm{C})$, and $67.2(\mathrm{C}-2,3,4,5), 74.2\left(\mathrm{OCH}_{2} \mathrm{Ph}\right)$, $70.4\left(\mathrm{OCH}_{2} \mathrm{CH}=\mathrm{CH}_{2}\right), 61.1(\mathrm{C}-6), 29.1$ and $19.0\left(\mathrm{C}\left(\mathrm{CH}_{3}\right) 2\right) ;{ }^{1} \mathrm{H}, \delta$ 7.37-7.28 (m, $\left.5 \mathrm{H}, \mathrm{Ph}\right), 5.930(\mathrm{~m}, 1 \mathrm{H}$, $\mathrm{OCH}_{2} \mathrm{CH}=\mathrm{CH}_{2}$ ), 5.34-5.20 (m, $\left.2 \mathrm{H}, \mathrm{OCH}_{2} \mathrm{CH}=\mathrm{CH}_{2}\right), 4.896$ and 4.759 (2 d, each $1 \mathrm{H}, \mathrm{OCH}_{2} \mathrm{Ph}$ ), 4.403 (d, $1 \mathrm{H}, \mathrm{H}-1), 4.355$ and 4.134 (2 m, each $1 \mathrm{H}, \mathrm{OCH}_{2} \mathrm{CH}=\mathrm{CH}_{2}$ ), 3.992 (dd, $\left.1 \mathrm{H}, \mathrm{H}-6 \mathrm{a}\right), 3.797$ (t, $1 \mathrm{H}, \mathrm{H}-6 \mathrm{~b}$ ), 3.721 ('t', 1 H, H-4), 3.538 (m, 1 H, H-2), 3.492 (t, 1 H, H-3), 3.264 (m, 1 H, H-5), 2.397 (d, 1 H, HO-2), 1.478 and $1.425\left(2 \mathrm{~s}\right.$, each $\left.3 \mathrm{H}, \mathrm{C}\left(\mathrm{CH}_{3}\right)_{2}\right) ; J_{1,2} 7.3, J_{2,3} 8.8, J_{3,4} 8.9, J_{4,5} 9.8, J_{5,6 \mathrm{a}} 5.4, J_{5,6 \mathrm{~b}} 10.5, J_{6 \mathrm{a}, 6 \mathrm{~b}}$ $-10.8, J_{2, \mathrm{OH}} 1.9 \mathrm{~Hz}$.

Anal. Calc. for $\mathrm{C}_{19} \mathrm{H}_{26} \mathrm{O}_{6}: \mathrm{C}, 65.13 ; \mathrm{H}, 7.48$. Found: $\mathrm{C}, 64.98 ; \mathrm{H}, 7.69$.

Allyl 2-O-acetyl-3-O-benzyl- $\beta$-D-glucopyranoside (6). - A solution of $4(5.7 \mathrm{~g}, 16.2 \mathrm{mmol}$ ) in pyridine $(55 \mathrm{~mL})$ and acetic anhydride $(30 \mathrm{~mL})$ was stirred for $20 \mathrm{~h}$, when TLC showed the disappearance of starting material and the formation of a new spot $\left(R_{\mathrm{F}} 0.52,9: 1 \mathrm{CH}_{2} \mathrm{Cl}_{2}\right.$-acetone). The solution was concentrated and coconcentrated with toluene $(2 \times 30 \mathrm{~mL})$, EtOH $(2 \times 30 \mathrm{~mL})$, and $\mathrm{CH}_{2} \mathrm{Cl}_{2}(2 \times 30 \mathrm{~mL})(\rightarrow 5$, quantitative). NMR data $\left(\mathrm{CDCl}_{3}\right):{ }^{13} \mathrm{C}, \delta 169.2\left(\mathrm{COCH}_{3}\right), 138.4$ and $128.1-127.4(\mathrm{Ph}), 133.4\left(\mathrm{OCH}_{2} \mathrm{CH}=\mathrm{CH}_{2}\right), 117.1$ $\left(\mathrm{OCH}_{2} \mathrm{CH}=\mathrm{CH}_{2}\right), 100.3(\mathrm{C}-1), 99.2\left(C\left(\mathrm{CH}_{3}\right)_{2}\right), 78.7,74.1,72.5$, and $66.9(\mathrm{C}-2,3,4,5), 73.6\left(\mathrm{OCH}_{2} \mathrm{Ph}\right)$, 
$69.7\left(\mathrm{OCH}_{2} \mathrm{CH}=\mathrm{CH}_{2}\right), 62.0(\mathrm{C}-6), 29.0$ and $18.9\left(\mathrm{C}\left(\mathrm{CH}_{3}\right) 2\right), 20.7\left(\mathrm{COCH}_{3}\right) ;{ }^{1} \mathrm{H}, \delta$ 7.34-7.27 (m, $\left.5 \mathrm{H}, \mathrm{Ph}\right)$, $5.819\left(\mathrm{~m}, 1 \mathrm{H}, \mathrm{OCH}_{2} \mathrm{CH}=\mathrm{CH}_{2}\right), 5.27-5.15\left(\mathrm{~m}, 2 \mathrm{H}, \mathrm{OCH}_{2} \mathrm{CH}=\mathrm{CH}_{2}\right), 4.987(\mathrm{t} t, 1 \mathrm{H}, \mathrm{H}-2), 4.811$ and 4.625 (2 d, each $1 \mathrm{H}, \mathrm{OCH}_{2} \mathrm{Ph}$ ), 4.452 (d, $\left.1 \mathrm{H}, \mathrm{H}-1\right), 4.290$ and 4.049 (2 m, each $1 \mathrm{H}, \mathrm{OCH}_{2} \mathrm{CH}_{2} \mathrm{CH}_{2}$ ), 3.932 (dd, 1 H, H-6a), 3.807 (t, 1 H, H-6b), 3.799 (t, 1 H, H-4), 3.547 (t, 1 H, H-3), 3.250 (m, 1 H, H-5), $2.001(\mathrm{~s}, 3 \mathrm{H}, \mathrm{Ac}), 1.493$ and $1.436\left(2 \mathrm{~s}\right.$, each $\left.3 \mathrm{H}, \mathrm{C}\left(\mathrm{CH}_{3}\right)_{2}\right) ; J_{1,2} 8.0, J_{2,3} 9.2, J_{3,4} 9.3, J_{4,5} 9.5, J_{5,6 \mathrm{a}} 5.4$, $J_{5,6 \mathrm{~b}} 10.5, J_{6 \mathrm{a}, 6 \mathrm{~b}}-10.8 \mathrm{~Hz}$.

To a solution of 5 in $\mathrm{MeOH}(100 \mathrm{~mL})$. Dowex-50 $\left(\mathrm{H}^{+}\right)$resin $(25 \mathrm{~g})$ was added, and the mixture was stirred for $2 \mathrm{~h}$ at $65^{\circ}$, when TLC showed the de- $O$-isopropylidenation to be complete $\left(R_{\mathrm{P}} 0.45 ; 4: 1 \mathrm{CH}_{2} \mathrm{Cl}_{2}\right.$ $\mathrm{MeOH})$. After filtration, the solution was evaporated to dryness, and column chromatography $\left(9: 1 \mathrm{CH}_{2} \mathrm{Cl}_{2}-\right.$ $\mathrm{MeOH})$ of the residue gave 6 , isolated as a white foam $(4.8 \mathrm{~g}, 84 \%)$, [ $\alpha]_{\mathrm{D}}-5^{\circ}\left(\mathrm{c} 1, \mathrm{CH}_{2} \mathrm{Cl}_{2}\right)$. ${ }^{1} \mathrm{H}-\mathrm{NMR}$ data $\left(\mathrm{CDCl}_{3}\right): \delta$ 7.34-7.29 (m, $\left.5 \mathrm{H}, \mathrm{Ph}\right), 5.844\left(\mathrm{~m}, 1 \mathrm{H}, \mathrm{OCH}_{2} \mathrm{CH}=\mathrm{CH}_{2}\right)$, 5.29-5.16 (m, $\left.2 \mathrm{H}, \mathrm{OCH}_{2} \mathrm{CH}=\mathrm{CH}_{2}\right)$, 4.994 (dd, $1 \mathrm{H}, \mathrm{H}-2), 4.746$ and 4.675 (2 d, each $1 \mathrm{H}, \mathrm{OCH}_{2} \mathrm{Ph}$ ), 4.462 (d, $\left.1 \mathrm{H}, \mathrm{H}-1\right), 4.308$ and 4.072 (2 $\mathrm{m}$, each $\left.1 \mathrm{H}, \mathrm{OCH}_{2} \mathrm{CH}=\mathrm{CH}_{2}\right), 3.701$ (m, $\left.1 \mathrm{H}, \mathrm{H}-4\right), 3.532$ (t, $\left.1 \mathrm{H}, \mathrm{H}-3\right), 3.357$ (m, $\left.1 \mathrm{H}, \mathrm{H}-5\right), 2.721$ (d, 1 H, HO-4), 2.027 (s, $3 \mathrm{H}, \mathrm{Ac}$ ); $J_{1,2} 8.0, J_{2,3} 9.5, J_{3,4} 9.2, J_{4,5} 9.5, J_{5,6 \mathrm{a}} 3.8, J_{5,6 \mathrm{~b}} 4.6, J_{4, \mathrm{OH}} 3.3 \mathrm{~Hz}$.

Anal. Calc. for $\mathrm{C}_{18} \mathrm{H}_{24} \mathrm{O}_{7} \cdot 0.5 \mathrm{H}_{2} \mathrm{O}: \mathrm{C}, 59.82 ; \mathrm{H}, 6.97$. Found: $\mathrm{C}, 59.97 ; \mathrm{H}, 6.96$.

Allyl 2-O-acetyl-3-O-benzyl-6-O-trityl- $\beta$-D-glucopyranoside (7).- A mixture of 6 (2.27 g, $6.44 \mathrm{mmol})$ and trityl chloride $(2.70 \mathrm{~g}, 9.7 \mathrm{mmol})$ in pyridine $(60 \mathrm{~mL})$ was stirred for $20 \mathrm{~h}$ at $90^{\circ}$. Then TLC showed the disappearance of the starting compound, and the formation of a new spot $\left(R_{\mathrm{F}} 0.27,95: 5 \mathrm{CH}_{2} \mathrm{Cl}_{2}\right.$-acetone). After concentration a solution of the residue in $\mathrm{CH}_{2} \mathrm{Cl}_{2}(30 \mathrm{~mL})$ was washed with water, $0.5 \mathrm{M}$ sulfuric acid, water, aq $10 \%$ sodium hydrogencarbonate, and water, dried $\left(\mathrm{MgSO}_{4}\right)$, filtered, and concentrated. Column chromatography $\left(95: 5 \mathrm{CH}_{2} \mathrm{Cl}_{2}\right.$-acetone) of the residue afforded 7 , isolated as a white solid $(3.00 \mathrm{~g}, 78 \%)$, $[\alpha]_{\mathrm{D}}$ $-12^{\circ}\left(c 1, \mathrm{CH}_{2} \mathrm{Cl}_{2}\right)$. NMR data $\left(\mathrm{CDCl}_{3}\right):{ }^{13} \mathrm{C}, \delta 169.4\left(\mathrm{COCH}_{3}\right), 143.5,138.1$, and 128.4-126.9 $(\mathrm{Ph}), 133.6$ $\left(\mathrm{OCH}_{2} \mathrm{CH}=\mathrm{CH}_{2}\right), 116.9\left(\mathrm{OCH}_{2} \mathrm{CH}=\mathrm{CH}_{2}\right), 99.6(\mathrm{C}-1), 86.7\left(\mathrm{OCPh}_{3}\right), 82.3,74.0,72.6$, and $71.6(\mathrm{C}-$ 2,3,4,5), 74.2 $\left(\mathrm{OCH}_{2} \mathrm{Ph}\right), 69.2\left(\mathrm{OCH}_{2} \mathrm{CH}=\mathrm{CH}_{2}\right), 63.6(\mathrm{C}-6), 20.7\left(\mathrm{COCH}_{3}\right) ;{ }^{1} \mathrm{H}, 8$ 7.47-7.23 (m, $20 \mathrm{H}, 4$ $\mathrm{Ph}$ ), $5.875\left(\mathrm{~m}, 1 \mathrm{H}, \mathrm{OCH}_{2} \mathrm{CH}=\mathrm{CH}_{2}\right), 5.30-5.16\left(\mathrm{~m}, 2 \mathrm{H}, \mathrm{OCH}_{2} \mathrm{CH}=\mathrm{CH}_{2}\right), 5.023$ (dd, $\left.1 \mathrm{H}, \mathrm{H}-2\right), 4.746$ and 4.699 ( $2 \mathrm{~d}$, each $\left.1 \mathrm{H}, \mathrm{OCH}_{2} \mathrm{Ph}\right), 4.436(\mathrm{~d}, 1 \mathrm{H}, \mathrm{H}-1), 4.37-4.30\left(\mathrm{~m}, 2 \mathrm{H}, \mathrm{OCH}_{2} \mathrm{CH}=\mathrm{CH}_{2}\right), 3.784(\mathrm{~m}, 1 \mathrm{H}$, H-4), 3.494 (t, $1 \mathrm{H}, \mathrm{H}-3$ ), 2.483 (d, $1 \mathrm{H}, \mathrm{HO}-4$ ), 2.024 (s, $3 \mathrm{H}, \mathrm{Ac}$ ); $J_{1,2} 8.0, J_{2,3} 9.5, J_{3,4} 9.2, J_{4, \mathrm{OH}} 2.7$ $\mathrm{Hz}$.

Anal. Calc. for $\mathrm{C}_{37} \mathrm{H}_{38} \mathrm{O}_{7} . \mathrm{H}_{2} \mathrm{O}: \mathrm{C}, 72.53 ; \mathrm{H}, 6.58$. Found: $\mathrm{C}, 72.42 ; \mathrm{H}, 6.38$.

2,3,4,6-Tetra-O-acetyl- $\alpha$-D-glucopyranosyl trichloroacetimidate (8). - A solution of 1,2,3,4,6-penta-Oacetyl- $\beta$-D-glucopyranose $(1.58 \mathrm{~g}, 4.05 \mathrm{mmol})$ in $N, N$-dimethylformamide $(5 \mathrm{~mL})$ was heated to $600^{\circ}$. To this solution was added hydrazine acetate $6(445 \mathrm{mg}, 4.8 \mathrm{mmol})$ and the mixture was stirred for $3 \mathrm{~h}$ at 600 . Then TLC (5:1 $\mathrm{CH}_{2} \mathrm{Cl}_{2}$-acetone) showed the complete disappearance of the starting compound and the formation of a new spot. The mixture was diluted with EtOAc $(10 \mathrm{~mL})$ and washed with aq $5 \%$ sodium chloride and water, dried $\left(\mathrm{MgSO}_{4}\right)$, filtered, and concentrated. To a solution of the residue in $\mathrm{CH}_{2} \mathrm{Cl}_{2}(20 \mathrm{~mL})$ at $00^{\circ}$, were added trichloroacetonitrile ( $4 \mathrm{~mL}, 40 \mathrm{mmol}$ ) and 1,8-diazabicyclo[5.4.0]undec-7-ene (0.75 mL, $5 \mathrm{mmol})$. After $2 \mathrm{~h}$ the starting material had disappeared (TLC; $5: 1 \mathrm{CH}_{2} \mathrm{Cl}_{2}$-acetone) and the solution was concentrated and purified by flash chromatography to yield $8(1.51 \mathrm{~g}, 76 \%)$, $[\alpha]_{\mathrm{D}}+18^{\circ}\left(c 1, \mathrm{CH}_{2} \mathrm{Cl}_{2}\right)$, lit ${ }^{16}[\alpha]_{578}^{20}+103^{\circ}(c 1.2$, $\left.\mathrm{CHCl}_{3}\right), R_{\mathrm{F}} 0.70\left(9: 1 \mathrm{CH}_{2} \mathrm{Cl}_{2}\right.$-acetone). NMR data $\left(\mathrm{CDCl}_{3}\right):{ }^{13} \mathrm{C}, \delta 169.9-169.0\left(\mathrm{COCH}_{3}\right), 160.1$ (OCNHCCl 3 ), $92.5(\mathrm{C}-1), 90.3\left(\mathrm{OCNHCCl}_{3}\right), 69.6,69.4,69.3$, and $67.4(\mathrm{C}-2,3,4,5), 61.0(\mathrm{C}-6), 20.2-$ $19.9\left(\mathrm{COCH}_{3}\right) ;{ }^{1} \mathrm{H}, \delta 8.699$ (s, $1 \mathrm{H}, \mathrm{OCNHCCl} 3$ ), 6.566 (d, $\left.1 \mathrm{H}, \mathrm{H}-1\right), 5.138$ (dd, $\left.1 \mathrm{H}, \mathrm{H}-2\right), 2.081,2.053$, 2.037, and $2.021\left(4 \mathrm{~s}\right.$, each $3 \mathrm{H}, 4 \mathrm{Ac}$ ); $J_{1,2} 3.7, J_{2,3} 10.2 \mathrm{~Hz}$. 
Allyl 2,3,6-tri-O-benzyl- $\beta$-D-galactopyranoside (10). - To a mixture of allyl 2,3-di-O-benzyl-4,6-O-benzylidene- $\beta$-D-galactopyranoside $(9,11.4 \mathrm{~g}, 23.3 \mathrm{mmol})$ and sodium cyanoborohydride $(19.0 \mathrm{~g}, 302 \mathrm{mmol})$ in dry tetrahydrofuran $(250 \mathrm{~mL})$ was added a saturated solution of hydrogen chloride in ether until the evolution of gas ceased. Then the mixture was diluted with $\mathrm{CH}_{2} \mathrm{Cl}_{2}(250 \mathrm{~mL})$ and water $(100 \mathrm{~mL})$, filtered through Celite, and the organic layer was washed with water, aq $10 \%$ sodium hydrogencarbonate, and water, dried $\left(\mathrm{MgSO}_{4}\right)$, filtered, and concentrated. Column chromatography $\left(95: 5 \mathrm{CH}_{2} \mathrm{Cl}_{2}\right.$-acetone) of the residue afforded 10, isolated as a syrup (6.82 $\mathrm{g}, 60 \%)$, $[\alpha]_{\mathrm{D}}+70\left(\mathrm{c} 1, \mathrm{CH}_{2} \mathrm{Cl}_{2}\right), \mathrm{RF}_{\mathrm{F}} 0.17$ (85:15 $\mathrm{CH}_{2} \mathrm{Cl}_{2}$-acetone). ${ }^{1} \mathrm{H}$-NMR data $\left(\mathrm{CDCl}_{3}\right)$ : S 7.36-7.25 (m, $15 \mathrm{H}, 3 \mathrm{Ph}), 5.951\left(\mathrm{~m}, 1 \mathrm{H}, \mathrm{OCH}_{2} \mathrm{CH}=\mathrm{CH}_{2}\right), 5.37-5.16\left(\mathrm{~m}, 2 \mathrm{H}, \mathrm{OCH}_{2} \mathrm{CH}=\mathrm{CH}\right), 4.928$ and 4.732 ( $2 \mathrm{~d}$, each $\left.1 \mathrm{H}, \mathrm{OCH}_{2} \mathrm{Ph}\right), 4.717$ and $4.586\left(2 \mathrm{~s}\right.$, each $\left.2 \mathrm{H}, 2 \mathrm{OCH}_{2} \mathrm{Ph}\right), 4.429$ and $4.136(2 \mathrm{~m}$, each $1 \mathrm{H}, \mathrm{OCH}_{2} \mathrm{CH}=\mathrm{CH}_{2}$ ), 4.413 (d, $\left.1 \mathrm{H}, \mathrm{H}-1\right), 4.018$ (m, $\left.1 \mathrm{H}, \mathrm{H}-4\right), 3.804$ (dd, $\left.1 \mathrm{H}, \mathrm{H}-6 \mathrm{a}\right), 3.723$ (dd, 1 H, H-6b), 3.680 (dd, 1 H, H-2), 3.553 (t, 1 H, H-5), 3.494 (dd, 1 H, H-3), 2.517 (bs, 1 H, HO-4); $J_{1,2} 7.8$, $J_{2,3} 9.4, J_{3,4} 3.4, J_{4,5}=0, J_{5,6 a} 5.9, J_{5,6 b} 6.0, J_{6 a, 6 b}-9.9 \mathrm{~Hz}$.

Anal. Calc. for $\mathrm{C}_{30} \mathrm{H}_{34} \mathrm{O}_{6}$ : C, 73.45; $\mathrm{H}, 6.99$. Found: $\mathrm{C}, 73.31 ; \mathrm{H}, 6.86$.

2,3-Di-O-benzyl-4,6-O-benzylidene-D-galactopyranose (11).- To a solution of allyl 2,3-di-O-benzyl$4,6-O$-benzylidene- $\beta$-D-galactopyranoside $8(9,1.72 \mathrm{~g}, 3.52 \mathrm{mmol})$ in $N, N$-dimethylformamide $(50 \mathrm{~mL})$ at $80^{\circ}$, was added potassium tert-butoxide until the solution turned black. After stirring for $2 \mathrm{~h}$ at $80^{\circ}$, the mixture was cooled to room temperature, diluted with $\mathrm{CH}_{2} \mathrm{Cl}_{2}(40 \mathrm{~mL})$, washed with water, and concentrated. The residue was dissolved in 9:1 acetone-water $(30 \mathrm{~mL})$, mercuric oxide $(14 \mathrm{mg})$ and mercuric chloride $(4.0 \mathrm{~g})$ were added, and the mixture was stirred for $2 \mathrm{~h}$ at room temperature, when TLC (7:3 hexane-EtOAc) showed the disappearance of starting material. After concentration, a solution of the residue in $\mathrm{CH}_{2} \mathrm{Cl}_{2}$ ( $40 \mathrm{~mL}$ ) was washed with water, aq $10 \%$ potassium iodide, and water, dried $\left(\mathrm{MgSO}_{4}\right)$, filtered, and concentrated. Column chromatography $\left(8: 2\right.$ hexane-EtOAc) of the residue afforded 11, isolated as a syrup $(1.18 \mathrm{~g}, 74 \%),[\alpha]_{\mathrm{D}}+70^{\circ}(c 1$, $\left.\mathrm{CH}_{2} \mathrm{Cl}_{2}\right)$, lit ${ }^{10}+78^{\circ}\left(c \mathrm{c} 0.5, \mathrm{CHCl}_{3}\right), R_{\mathrm{F}} 0.32\left(7: 3\right.$ hexane-EtOAc). NMR data $\left(\mathrm{CDCl}_{3}\right):{ }^{13} \mathrm{C}, \delta 138.4-137.7$ and 128.9-125.6 (Ph), $100.7(\mathrm{OCHPh}), 97.2(\mathrm{C}-1 \beta), 92.0(\mathrm{C}-1 \alpha) ;{ }^{1} \mathrm{H}, \delta$ 7.37-7.26 (m, $\left.15 \mathrm{H}, 3 \mathrm{Ph}\right), 5.503$ and $5.491(2 \mathrm{~s}, 1 \mathrm{H}, \mathrm{OCHPh}), 5.373(\mathrm{~d}, 0.5 \mathrm{H}, \mathrm{H}-1 \alpha) ; J_{1 \alpha, 2} 3.4 \mathrm{~Hz}$.

2,3-Di-O-benzyl-4,6-O-benzylidene- $\alpha$-D-galactopyranosyl trichloroacetimidate (12). - To a solution of 11 (623 mg, $1.39 \mathrm{mmol}$ ) and trichloroacetonitrile $(1.4 \mathrm{~mL}, 14 \mathrm{mmol})$ in $\mathrm{CH}_{2} \mathrm{Cl}_{2}$ (15 mL) was added 1,8-diazabicyclo[5.4.0]undec-7-ene ( $250 \mu \mathrm{L}, 1.67 \mathrm{mmol})$. After stirring for $2 \mathrm{~h}, \mathrm{TLC}$ (85:15 $\mathrm{CH}_{2} \mathrm{Cl}_{2}$-acetone) showed the disappearance of starting compound. The solution was concentrated and purified by flash chromatography to yield 12 as a glass $(793 \mathrm{mg}, 96 \%), R_{\mathrm{F}} 0.27$ (95:5 toluene-acetone). ${ }^{1} \mathrm{H}-\mathrm{NMR}$ data $\left(\mathrm{CDCl}_{3}\right): \delta$ $8.561\left(\mathrm{~s}, 1 \mathrm{H}, \mathrm{OCN} H \mathrm{CCl}_{3}\right), 7.52-7.26(\mathrm{~m}, 15 \mathrm{H}, 3 \mathrm{Ph}), 6.635(\mathrm{~d}, 1 \mathrm{H}, \mathrm{H}-1), 5.511(\mathrm{~s}, 1 \mathrm{H}, \mathrm{OCHPh}) ; J_{1,2}$ $3.4 \mathrm{~Hz}$.

Allyl 4-0-(2,3-di-0-benzyl-4,6-0-benzylidene- $\beta$-D-glucopyranosyl)-2,3,6-tri-O-benzyl- $\beta$-D-glucopyranoside (16). - A solution of allyl 4-O-(2,3,4,6-tetra-O-acetyl- $\beta$-D-glucopyranosyl)-2,3,6-tri-O-acetyl- $\beta$-D-glucopyranoside ${ }^{11}(13,10.8 \mathrm{~g}, 16 \mathrm{mmol})$ in $\mathrm{CH}_{2} \mathrm{Cl}_{2}(25 \mathrm{~mL})$ and $\mathrm{MeOH}(80 \mathrm{~mL})$ was adjusted to $\mathrm{pH} 10$ by the addition of sodium methoxide, stirred overnight, and then neutralised with Dowex-50 $\left(\mathrm{H}^{+}\right)$resin, filtered, and concentrated ( $\rightarrow 14$, quantitative). To a solution of the residue in $N, N$-dimethylformamide $(35 \mathrm{~mL})$ were added benzaldehyde dimethyl acetal $(3.6 \mathrm{~mL}, 24 \mathrm{mmol}$ ) and a catalytic amount of p-toluenesulfonic acid, and the mixture was stirred under reduced pressure for $2 \mathrm{~h}$ at $50^{\circ}$, neutralised with solid sodium hydrogencarbonate, filtered, and concentrated $(\rightarrow 15)$. A solution of the crude residue and benzyl bromide $(14.5 \mathrm{~mL}, 122 \mathrm{mmol})$ in $N, N$-dimethylformamide $(75 \mathrm{~mL})$ was added to a suspension of sodium hydride $(3.2 \mathrm{~g}, 133 \mathrm{mmol})$ in $N, N$ dimethylformamide $(80 \mathrm{~mL})$ at $0^{\circ}$. After stirring overnight, $\mathrm{MeOH}(25 \mathrm{~mL})$ was added, and the solution was diluted with $\mathrm{CH}_{2} \mathrm{Cl}_{2}(150 \mathrm{~mL})$, washed with water, dried $\left(\mathrm{MgSO}_{4}\right)$, filtered, and concentrated. Column chro- 
matography (93:7 toluene-acetone) of the residue afforded 16 , isolated as a white solid $(11.4 \mathrm{~g}, 77 \%),[\alpha]_{\mathrm{D}}$ $+4^{\circ}\left(\mathrm{c} 1, \mathrm{CH}_{2} \mathrm{Cl}_{2}\right), R_{\mathrm{F}} 0.43$ (9:1 toluene-acetone). ${ }^{1} \mathrm{H}-\mathrm{NMR}$ data $\left(\mathrm{CDCl}_{3}\right): \delta$ 7.39-7.24 (m, $\left.30 \mathrm{H}, 6 \mathrm{Ph}\right)$, $5.954\left(\mathrm{~m}, 1 \mathrm{H}, \mathrm{OCH}_{2} \mathrm{CH}=\mathrm{CH}_{2}\right), 5.486(\mathrm{~s}, 1 \mathrm{H}, \mathrm{OCHPh}), 5.335$ and $5.290\left(2 \mathrm{~m}\right.$, each $\left.1 \mathrm{H}, \mathrm{OCH}_{2} \mathrm{CH}_{2} \mathrm{CH}_{2}\right)$.

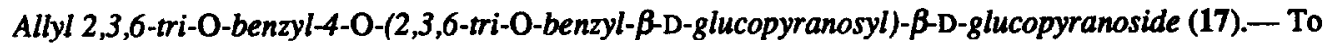
a suspension of $16(9.9 \mathrm{~g}, 10.7 \mathrm{mmol})$ and sodium cyanoborohydride $(7.4 \mathrm{~g}, 118 \mathrm{mmol})$ in freshly distilled tetrahydrofuran $(150 \mathrm{~mL})$ was added a saturated solution of hydrogen chloride in ether until the evolution of gas ceased (pH 3). Then the mixture was diluted with $\mathrm{CH}_{2} \mathrm{Cl}_{2}(200 \mathrm{~mL})$, and the solution was filtered through silica, washed with water, aq $10 \%$ sodium hydrogencarbonate, and water, dried $\left(\mathrm{MgSO}_{4}\right)$, filtered, and concentrated. Column chromatography (95:5 toluene-acetone) of the residue gave 17, isolated as a syrup (7.74 $\mathrm{g}$, $78 \%),[\alpha]_{\mathrm{D}}+17^{\circ}\left(\mathrm{c} 1, \mathrm{CH}_{2} \mathrm{Cl}_{2}\right), R_{\mathrm{F}} 0.36$ (9:1 toluene-acetone). $\mathrm{NMR}$ data $\left(\mathrm{CDCl}_{3}\right):{ }^{13} \mathrm{C}, \delta$ 138.9-137.8 and 127.9-126.7 (Ph), 133.8 $\left(\mathrm{OCH}_{2} \mathrm{CH}=\mathrm{CH}_{2}\right), 116.6\left(\mathrm{OCH}_{2} \mathrm{CH}=\mathrm{CH}_{2}\right), 102.3$ and $\left.102.0(\mathrm{C}-1,1)^{\prime}\right), 84.1,82.4$, 81.8, 81.3, 76.2, 74.7, 73.5, and $72.4\left(\mathrm{C}-2,3,4,5,2^{\prime}, 3^{\prime}, 4^{\prime}, 5^{\prime}\right), 74.8,74.6,74.5(2 \mathrm{C}), 73.1$, and $72.8(6$ $\left.\mathrm{OCH}_{2} \mathrm{Ph}\right), 70.4\left(\mathrm{OCH}_{2} \mathrm{CH}=\mathrm{CH}_{2}\right), 69.7$ and $67.8\left(\mathrm{C}-6,6^{\prime}\right) ;{ }^{1} \mathrm{H}, \delta$ 7.34-7.20 (m, $\left.30 \mathrm{H}, 6 \mathrm{Ph}\right), 5.949(\mathrm{~m}, 1 \mathrm{H}$, $\left.\mathrm{OCH}_{2} \mathrm{CH}=\mathrm{CH}_{2}\right), 5.326$ and $5.192\left(2 \mathrm{~m}\right.$, each $\left.1 \mathrm{H}, \mathrm{OCH}_{2} \mathrm{CH}=\mathrm{CH}_{2}\right), 4.119\left(\mathrm{~m}, 1 \mathrm{H}, \mathrm{OCH} H \mathrm{CH}=\mathrm{CH}_{2}\right), 2.878$ (d, $1 \mathrm{H}, \mathrm{HO}-4$ ').

Anal. Calc. for $\mathrm{C}_{57} \mathrm{H}_{62} \mathrm{O}_{11}: \mathrm{C}, 74.2 ; \mathrm{H}, 6.8$. Found: $\mathrm{C}, 73.9 ; \mathrm{H}, 6.8$.

4-O-(2,3-Di-O-benzyl-4,6-O-benzylidene- $\beta$-D-glucopyranosyl)-2,3,6-tri-O-benzyl-D-glucopyranosyl trichloroacetimidate (19). - To a solution of $16(1.41 \mathrm{~g}, 1.53 \mathrm{mmol})$ in $N, N$-dimethylformamide $(50 \mathrm{~mL})$ at $80^{\circ}$ was added potassium tert-butoxide. After $2 \mathrm{~h}$ the mixture was cooled, diluted with $\mathrm{CH}_{2} \mathrm{Cl}_{2}(50 \mathrm{~mL})$, and the solution was washed with water, dried $\left(\mathrm{MgSO}_{4}\right)$, filtered, and concentrated. The residue was dissolved in 9:1 acetone-water $(100 \mathrm{~mL})$, and mercuric oxide $(17 \mathrm{mg})$ and mercuric chloride $(2.4 \mathrm{~g})$ were added. After stirring overnight, the mixture was concentrated, and the residue dissolved in $\mathrm{CH}_{2} \mathrm{Cl}_{2}(75 \mathrm{~mL})$. The solution was washed with water, aq $5 \%$ potassium iodide, water, aq $10 \%$ sodium hydrogencarbonate, and water, dried $\left(\mathrm{MgSO}_{4}\right)$, filtered, and concentrated, affording $18(1.25 \mathrm{~g}, 93 \%)$. To a solution of $18(0.8 \mathrm{~g}, 0.91 \mathrm{mmol})$ in $\mathrm{CH}_{2} \mathrm{Cl}_{2}(25 \mathrm{~mL})$ were added trichloroacetonitrile $(0.9 \mathrm{~mL}, 9.0 \mathrm{mmol})$ and 1,8-diazabicyclo[5.4.0]undec-7-ene $(160 \mu \mathrm{L}, 1.1 \mathrm{mmol})$. After stirring for $2 \mathrm{~h}$, TLC (7:3 hexane-EtOAc) showed a complete conversion of 18, and the mixture was concentrated and purified by flash chromatography (7:3 hexane-EtOAc) to yield 19, isolated as a syrup (736 mg, $79 \%)$, [ $\left.\alpha]_{\mathrm{D}}+112^{\circ}(c), \mathrm{CH}_{2} \mathrm{Cl}_{2}\right), R_{\mathrm{F}} 0.54$ (7:3 hexane-EtOAc). ${ }^{1} \mathrm{H}-\mathrm{NMR}$ data $\left(\mathrm{CDCl}_{3}\right): \delta$ $8.699\left(\mathrm{~s}, 0.7 \mathrm{H}, \alpha-\mathrm{OCN} H \mathrm{CCl}_{3}\right), 8.590\left(\mathrm{~s}, 0.3 \mathrm{H}, \beta-\mathrm{OCN} H \mathrm{CCl}_{3}\right), 6.439$ (d, $0.3 \mathrm{H}, \mathrm{H}-1 \alpha$ ), 5.781 (d, $0.7 \mathrm{H}$, $\mathrm{H}-1 \beta$ ), 5.499 (s, $0.3 \mathrm{H}, \alpha-\mathrm{OCHPh}), 5.480(\mathrm{~s}, 0.7 \mathrm{H}, \beta-\mathrm{OCHPh}) ; J_{1 \alpha, 2} 3.3, J_{1 \beta, 2} 7.5 \mathrm{~Hz}$.

Allyl 2-O-acetyl-3-O-benzyl-4-O-(2,3-di-O-benzyl-4,6-0-benzylidene- $\alpha$-D-galactopyranosyl)-6-0-trityl$\beta$-D-glucopyranoside (20). - A mixture of $7(320 \mathrm{mg}, 538 \mu \mathrm{mol}), 12(360 \mathrm{mg}, 607 \mu \mathrm{mol})$ and powdered molecular sieves $(4 \AA, 1 \mathrm{~g})$ in $\mathrm{CH}_{2} \mathrm{Cl}_{2}(2 \mathrm{~mL})$ and ether $(15 \mathrm{~mL})$ was stirred and cooled to $-70^{\circ}$. Then a solution of trimethylsilyl trifluoromethanesulfonate $(10 \mu \mathrm{L}, 55 \mu \mathrm{mol})$ in ether $(100 \mu \mathrm{L})$ was added and stirring was continued for $16 \mathrm{~h}$, when TLC showed the disappearance of 7 and the formation of a new spot $\left(R_{F} 0.43,95: 5\right.$ toluene-acetone). Triethyl amine was added, and the mixture was filtered through Celite, washed with water, aq $10 \%$ sodium hydrogencarbonate, and water, dried $\left(\mathrm{MgSO}_{4}\right)$, filtered, and concentrated. Column chromatography (95:5 toluene-acetone) of the residue afforded 20, isolated as an amorphous powder $(314 \mathrm{mg}, 60 \%)$, $[\alpha]_{D}$ $+128^{\circ}\left(c 1, \mathrm{CH}_{2} \mathrm{Cl}_{2}\right)$. NMR data $\left(\mathrm{CDCl}_{3}\right):{ }^{13} \mathrm{C}, \delta 169.3\left(\mathrm{COCH}_{3}\right), 143.5,138.5-137.6$, and 128.6-126.0 (Ph), $133.5\left(\mathrm{OCH}_{2} \mathrm{CH}=\mathrm{CH}_{2}\right), 117.0\left(\mathrm{OCH}_{2} \mathrm{CH}=\mathrm{CH}_{2}\right), 100.4(\mathrm{OCHPh}), 99.3(\mathrm{C}-1), 96.4\left(\mathrm{C}-1^{\prime}\right), 86.3$ (OCPh3), 83.8, 76.0, 74.5, 74.1, 73.6, 72.7, 70.2, and 62.5 (C-2,3,4,5,2',3',4',5'), 73.8 and 71.4 (2 C) (3 $\left.\mathrm{OCH}_{2} \mathrm{Ph}\right), 69.0$ and $68.9\left(\mathrm{C}-6{ }^{\prime}\right.$ and $\left.\mathrm{OCH}_{2} \mathrm{CH}=\mathrm{CH}_{2}\right), 63.3(\mathrm{C}-6), 20.6\left(\mathrm{COCH}_{3}\right) ;{ }^{1} \mathrm{H}, \delta$ 7.47-7.13 (m, $35 \mathrm{H}$, $7 \mathrm{Ph}), 5.929\left(\mathrm{~m}, 1 \mathrm{H}, \mathrm{OCH}_{2} \mathrm{CH}=\mathrm{CH}_{2}\right), 5.719(\mathrm{~d}, 1 \mathrm{H}, \mathrm{H}-1), 5.431(\mathrm{~s}, 1 \mathrm{H}, \mathrm{OCHPh}), 5.321$ and $5.224(2$ 
$\mathrm{m}$, each $\left.1 \mathrm{H}, \mathrm{OCH}_{2} \mathrm{CH}=\mathrm{CH}_{2}\right), 5.167$ (dd, $\left.1 \mathrm{H}, \mathrm{H}-2\right), 4.76-4.53\left(\mathrm{~m}, 6 \mathrm{H}, 3 \mathrm{OCH}_{2} \mathrm{Ph}\right), 4.532(\mathrm{~d}, 1 \mathrm{H}, \mathrm{H}-1)$, 4.458 and $4.208\left(2 \mathrm{~m}\right.$, each $1 \mathrm{H}, \mathrm{OCH}_{2} \mathrm{CH}=\mathrm{CH}_{2}$ ), 4.100 (dd, $\left.1 \mathrm{H}, \mathrm{H}-4\right), 3.951$ (dd, $1 \mathrm{H}, \mathrm{H}-2$ ), 3.941 (dd, 1 H, H-6b'), 3.824 (t, $1 \mathrm{H}, \mathrm{H}-3$ ), 3.802 (d, $1 \mathrm{H}, \mathrm{H}-\mathbf{4}^{\prime}$ ), 3.676 (m, $1 \mathrm{H}, \mathrm{H}-5$ ), 3.516 (dd, $\left.1 \mathrm{H}, \mathrm{H}-6 \mathrm{a}^{\prime}\right), 3.501$ (dd, 1 H, H-3'), 3.417 (dd, 1 H, H-6b), 3.286 (dd, 1 H, H-6a), 3.010 (bs, 1 H, H-5'), 1.918 (s, 3 H, Ac); $J_{1,2} 7.8, J_{2,3} 9.3, J_{3,4} 8.5, J_{4,5} 9.3, J_{5,6 \mathrm{a}} 2.1, J_{5,6 \mathrm{~b}} 5.3, J_{6 \mathrm{a}, 6 \mathrm{~b}}-9.7, J_{1^{\prime}, 2^{\prime}} 3.7, J_{2^{\prime}, 3^{\prime}} 10.2, J_{3^{\prime}, 4^{\prime}} 3.3$,

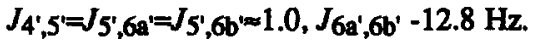

Allyl 2-O-acetyl-3-O-benzyl-4-0-(2,3-di-O-benzyl-4,6-O-benzylidene- $\alpha$-D-galactopyranosyl)- $\beta$-D-glucopyranoside (21). - To a solution of $20(84 \mathrm{mg}, 82 \mu \mathrm{mol})$ in $\mathrm{CH}_{2} \mathrm{Cl}_{2}(5 \mathrm{~mL})$ were added a few drops of aq $70 \%$ perchloric acid, whereby a deep yellow colour appeared. The mixture was neutralised with solid sodium hydrogencarbonate, filtered through Celite, washed with water, dried $\left(\mathrm{MgSO}_{4}\right)$, filtered, and concentrated. Column chromatography (95:5 toluene-acetone) of the residue gave 21 , isolated as a syrup (49 mg, 76\%), [ $\alpha]_{D}$ $+104^{\circ}\left(c\right.$ 1, $\mathrm{CH}_{2} \mathrm{Cl}_{2}$ ), $R_{\mathrm{F}} 0.23$ (95:5 toluene-acetone). ${ }^{1} \mathrm{H}-\mathrm{NMR}$ data $\left(\mathrm{CDCl}_{3}\right): 87.45-7.15(\mathrm{~m}, 20 \mathrm{H}, 4 \mathrm{Ph}$ ), $5.839\left(\mathrm{~m}, 1 \mathrm{H}, \mathrm{OCH}_{2} \mathrm{CH}=\mathrm{CH}_{2}\right), 5.661\left(\mathrm{~d}, 1 \mathrm{H}, \mathrm{H}-1^{\prime}\right), 5.467(\mathrm{~s}, 1 \mathrm{H}, \mathrm{OCHPh}), 5.249$ and 5.174 (2 m, each $1 \mathrm{H}, \mathrm{OCH}_{2} \mathrm{CH}=\mathrm{CH}_{2}$ ), 5.026 (dd, $\left.1 \mathrm{H}, \mathrm{H}-2\right), 4.83-4.53\left(\mathrm{~m}, 6 \mathrm{H}, 3 \mathrm{OCH}_{2} \mathrm{Ph}\right), 4.459$ (d, $\left.1 \mathrm{H}, \mathrm{H}-1\right), 4.294$ $\left(\mathrm{m}, 1 \mathrm{H}, \mathrm{OCH} H \mathrm{CH}=\mathrm{CH}_{2}\right), 3.493(\mathrm{~m}, 1 \mathrm{H}, \mathrm{H}-5), 1.866(\mathrm{~s}, 3 \mathrm{H}, \mathrm{Ac}) ; J_{1,2} 7.8, J_{2,3} 9.2, J_{4,5} 9.5, J_{5,6 \mathrm{a}} 2.5$, $J_{5,6 \mathrm{~b}} 4.4, J_{1,2}, 3.7 \mathrm{~Hz}$.

Methyl [allyl 2-O-acetyl-3-0-benzyl-4-O-(2,3-di-O-benzyl-4,6-O-benzylidene- $\alpha$-D-galactopyranosyl)- $\beta$ D-glucopyranosid] uronate (23). - To a solution of $21(59 \mathrm{mg}, 75.4 \mu \mathrm{mol})$ in acetone ( $5 \mathrm{~mL}$ ) at 00 was added Jones reagent $(100 \mu \mathrm{L})$, and the mixture was stirred for $5 \mathrm{~h}$ while the temperature was allowed to rise to room temperature. The mixture was poured onto ice and extracted with $\mathrm{CH}_{2} \mathrm{Cl}_{2}(3 \times 5 \mathrm{~mL})$. The combined extracts were washed with water, dried $\left(\mathrm{MgSO}_{4}\right)$, filtered, and concentrated $(\rightarrow 22)$. To a solution of the crude residue in $\mathrm{CH}_{2} \mathrm{Cl}_{2}(5 \mathrm{~mL})$ was added dropwise a solution of diazomethane in ether until the yellow colour persisted. The excess of diazomethane was destroyed with acetic acid and the mixture was poured in water $(5 \mathrm{~mL})$. The layers were separated and the organic layer was washed with water, aq 10\% sodium hydrogencarbonate, and water, dried $\left(\mathrm{MgSO}_{4}\right)$, filtered, and concentrated. Column chromatography (98:2 toluene-acetone) of the residue afforded 23 , isolated as a syrup $(43 \mathrm{mg}, 70 \%)$, [ $\alpha]_{\mathrm{D}}+126^{\circ}\left(\mathrm{c} 1, \mathrm{CH}_{2} \mathrm{Cl}_{2}\right), R_{\mathrm{F}} 0.31$ (95:5 toluene-acetone). NMR data $\left(\mathrm{CDCl}_{3}\right):{ }^{13} \mathrm{C}, \delta 169.2$ and $168.3\left(\mathrm{C}-6\right.$ and $\left.\mathrm{COCH}_{3}\right), 138.5-137.7$ and 128.8-126.2 (Ph), $133.2\left(\mathrm{OCH}_{2} \mathrm{CH}=\mathrm{CH}_{2}\right), 117.3\left(\mathrm{OCH}_{2} \mathrm{CH}=\mathrm{CH}_{2}\right), 100.8(\mathrm{OCHPh}), 99.7$ and $99.5\left(\mathrm{C}-1,1^{\prime}\right), 81.1,78.1,75.5$, 75.2, 75.1, 74.4, 72.2, and $63.3\left(\mathrm{C}-2,3,4,5,2^{\prime}, 3^{\prime}, 4^{\prime}, 5^{\prime}\right), 74.3,73.6,71.6,69.6$, and $69.1\left(3 \mathrm{OCH}_{2} \mathrm{Ph}\right.$, $\mathrm{OCH}_{2} \mathrm{CH}=\mathrm{CH}_{2}$, and $\left.\mathrm{C}-6^{\prime}\right), 52.6\left(\mathrm{OCH}_{3}\right), 20.6\left(\mathrm{COCH}_{3}\right) ;{ }^{1} \mathrm{H}, 87.42-7.07(\mathrm{~m}, 20 \mathrm{H}, 4 \mathrm{Ph}), 5.723(\mathrm{~m}, 1 \mathrm{H}$, $\left.\mathrm{OCH}_{2} \mathrm{CH}=\mathrm{CH}_{2}\right), 5.394(\mathrm{~s}, 1 \mathrm{H}, \mathrm{OCH} \mathrm{Ph}), 5.253\left(\mathrm{~d}, 1 \mathrm{H}, \mathrm{H}-1^{\prime}\right), 5.156$ and $5.084(2 \mathrm{~m}$, each $1 \mathrm{H}$, $\mathrm{OCH}_{2} \mathrm{CH}=\mathrm{CH}_{2}$ ), 4.953 (dd, $\left.1 \mathrm{H}, \mathrm{H}-2\right), 4.80-4.44$ (m, $6 \mathrm{H}, 3 \mathrm{OCH}_{2} \mathrm{Ph}$ ), 4.378 (d, $\left.1 \mathrm{H}, \mathrm{H}-1\right), 4.216$ (m, 1 $\left.\mathrm{H}, \mathrm{OCH} H \mathrm{CH}=\mathrm{CH}_{2}\right), 3.648\left(\mathrm{~s}, 3 \mathrm{H}, \mathrm{OCH}_{3}\right), 3.592(\mathrm{t}, 1 \mathrm{H}, \mathrm{H}-3), 3.441$ (bs, $\left.1 \mathrm{H}, \mathrm{H}-5^{\prime}\right), 1.782(\mathrm{~s}, 3 \mathrm{H}, \mathrm{Ac})$; $J_{1,2} 7.5, J_{2,3} 9.0, J_{3,4} 8.7, J_{1,2} \cdot 3.5 \mathrm{~Hz}$.

Allyl 3-O-benzyl-4-O-(2,3-di-O-benzyl-4,6-0-benzylidene- $\alpha$-D-galactopyranosyl)- $\beta$-D-glucopyranosiduranic acid (24). - To a solution of $23(42 \mathrm{mg}, 51.8 \mu \mathrm{mol})$ in $\mathrm{CH}_{2} \mathrm{Cl}_{2}(1 \mathrm{~mL})$ and $\mathrm{MeOH}(5 \mathrm{~mL})$ was added sodium methoxide ( $\mathrm{pH} 11$ ). After stirring for $20 \mathrm{~h}$, the solution was neutralised with Dowex-50 $\left(\mathrm{H}^{+}\right)$resin, filtered, and concentrated. A solution of the residue in acetone $(3 \mathrm{~mL})$ and $1 \mathrm{M}$ lithium hydroxide $(1 \mathrm{~mL})$ was stirred for $3 \mathrm{~h}$, when TLC showed the reaction to be complete. Then the solution was filtered through a column of Dowex-50 $\left(\mathrm{H}^{+}\right)$resin, concentrated, and co-concentrated with toluene $(3 \times 3 \mathrm{~mL}), \mathrm{EtOH}(3 \times 3 \mathrm{~mL})$, and $\mathrm{CH}_{2} \mathrm{Cl}_{2}(3 \times 3 \mathrm{~mL})$ to afford 24 as a foam $(35 \mathrm{mg}, 90 \%)$, $[\alpha]_{\mathrm{D}}+106^{\circ}\left(c 1, \mathrm{CH}_{2} \mathrm{Cl}_{2}\right), R_{\mathrm{F}} 0.22$ (95:5 tolueneacetone). ${ }^{1} \mathrm{H}-\mathrm{NMR}$ data $\left(\mathrm{CDCl}_{3}\right): \delta$ 7.36-7.21 (m, $\left.20 \mathrm{H}, 4 \mathrm{Ph}\right), 5.907\left(\mathrm{~m}, 1 \mathrm{H}, \mathrm{OCH}_{2} \mathrm{CH}=\mathrm{CH}_{2}\right), 5.453(\mathrm{~s}, 1$ $\mathrm{H}, \mathrm{OCHPh})$, 5.37-5.19 (m, $\left.2 \mathrm{H}, \mathrm{OCH}_{2} \mathrm{CH}=\mathrm{CH}_{2}\right), 5.178\left(\mathrm{~d}, 1 \mathrm{H}, \mathrm{H}-1^{\prime}\right) ; J_{1 ', 2} 3.6 \mathrm{~Hz}$. 
Propyl 4-O- $\alpha$-D-galactopyranosyl- $\beta$-D-glucopyranosiduronic acid (25).- A solution of 24 (35 mg, 46.4 $\mu \mathrm{mol})$ in EtOAc $(2 \mathrm{~mL})$ and $\mathrm{EtOH}(5 \mathrm{~mL})$, containing $10 \% \mathrm{Pd}-\mathrm{C}(20 \mathrm{mg})$, was hydrogenolysed at $1 \mathrm{~kg} / \mathrm{cm}^{2}$ for $8 \mathrm{~h}$, when TLC (4:1 $\left.\mathrm{CH}_{2} \mathrm{Cl}_{2}-\mathrm{MeOH}\right)$ showed the absence of $\mathrm{UV}$-positive spots. The mixture was filtered through Celite, and concentrated. Purification on a Sep-Pak C-18 cartridge $\left(\mathrm{H}_{2} \mathrm{O}\right)$ and lyophilisation gave 25, isolated as a white powder $(13.5 \mathrm{mg}, 73 \%),[\alpha]_{\mathrm{D}}+98^{\circ}(c 1$, water $), R_{\mathrm{F}} 0.34\left(1: 1 \mathrm{CH}_{2} \mathrm{Cl}_{2}-\mathrm{MeOH}\right)$. NMR data $\left(\mathrm{D}_{2} \mathrm{O}\right):{ }^{13} \mathrm{C}, \delta 174.5(\mathrm{C}-6), 104.6(\mathrm{C}-1), 101.3(\mathrm{C}-1), 78.8,78.3,76.4,75.1,73.3,71.5,71.1$, and $70.6(\mathrm{C}-$ 2,3,4,5,2', 3',4',5'), $74.0\left(\mathrm{OCH}_{2} \mathrm{CH}_{2} \mathrm{CH}_{3}\right), 62.6\left(\mathrm{C}^{\prime} 6^{\prime}\right), 24.5\left(\mathrm{OCH}_{2} \mathrm{CH}_{2} \mathrm{CH}_{3}\right), 11.9\left(\mathrm{OCH}_{2} \mathrm{CH}_{2} \mathrm{CH}_{3}\right) ;{ }^{1} \mathrm{H}$ (COSY, HOHAHA), $\delta 5.482$ (d, 1 H, H-1'), 4.539 (d, 1 H, H-1), 4.097 (d, 1 H, H-5), 3.991 (H-4'), 3.852 and $\left.3.614\left(\mathrm{OCH}_{2} \mathrm{CH}_{2} \mathrm{CH}_{3}\right), 3.811(\mathrm{H}-4), 3.802(\mathrm{H}-2), 3.764(\mathrm{H}-3), 3.345(\mathrm{H}-3)^{\prime}\right), 3.329(\mathrm{H}-2), 1.614(\mathrm{~m}$, $\left.2 \mathrm{H}, \mathrm{OCH}_{2} \mathrm{CH}_{2} \mathrm{CH}_{3}\right), 0.898\left(\mathrm{t}, 3 \mathrm{H}, \mathrm{OCH}_{2} \mathrm{CH}_{2} \mathrm{CH}_{3}\right) ; J_{1,2} 8.0, J_{4,5} 9.2, J_{1} ; 2,3.2 \mathrm{~Hz}$.

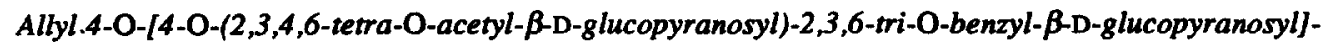
2,3,6-tri-O-benzyl- $\beta$-D-glucopyranoside (26). - A mixture of $17(693 \mathrm{mg}, 0.92 \mathrm{mmol})$, powdered molecular sieves $(4 \AA, 1.5 \mathrm{~g})$, and trimethylsilyl trifluoromethanesulfonate $(200 \mu \mathrm{L}, 1.2 \mathrm{mmol})$ in $\mathrm{CH}_{2} \mathrm{Cl}_{2}(5 \mathrm{~mL})$ was cooled to $-30^{\circ}$ and stirred for $20 \mathrm{~min}$. Then a solution of $8(600 \mathrm{mg}, 1.22 \mathrm{mmol})$ in $\mathrm{CH}_{2} \mathrm{Cl}_{2}(5 \mathrm{~mL})$ was added dropwise. After stirring for $16 \mathrm{~h}$, TLC showed the disappearance of 17 and the formation of a new product (85:15 $\mathrm{CH}_{2} \mathrm{Cl}_{2}$-acetone; $26 R_{\mathrm{F}} 0.42$ ), and triethyl amine (1.5 mL) was added. The mixture was filtered and concentrated, and a solution of the residue in $\mathrm{CH}_{2} \mathrm{Cl}_{2}(10 \mathrm{~mL})$ was washed with water, dried $\left(\mathrm{MgSO}_{4}\right)$, filtered, and concentrated. Column chromatography (9:1 toluene-acetone) of the residue afforded 26, isolated as a syrup (695 mg, 74\%), $[\alpha]_{\mathrm{D}}-2^{\circ}\left(c 1, \mathrm{CH}_{2} \mathrm{Cl}_{2}\right)$. NMR data $\left(\mathrm{CDCl}_{3}\right):{ }^{13} \mathrm{C}, \delta 170.5,170.1,169.2$, and 168.9 (4 $\left.\mathrm{COCH}_{3}\right), 139.2-137.9$ and 128.4-126.9 $(\mathrm{Ph}), 133.9\left(\mathrm{OCH}_{2} \mathrm{CH}=\mathrm{CH}_{2}\right), 117.1\left(\mathrm{OCH}_{2} \mathrm{CH}=\mathrm{CH} 2\right), 102.5$, 102.3, and 99.7 (C-1,1',1"), 82.8 (2 C), 81.8, 81.5, 76.8 (2 C), 74.7, 74.5, 72.9, 71.6, 71.3, and 68.0 $\left(\mathrm{C}-2,3,4,5,2^{\prime}, 3^{\prime}, 4^{\prime}, 5^{\prime}, 2^{\prime \prime}, 3^{\prime \prime}, 4^{\prime \prime}, 5^{\prime \prime}\right), 75.0,74.8$ (3 C), 73.1, and 73.0 (6 $\left.\mathrm{OC} \mathrm{H}_{2} \mathrm{Ph}\right), 70.1$ $\left(\mathrm{OCH}_{2} \mathrm{CH}=\mathrm{CH}_{2}\right), 67.9$ and $67.5\left(\mathrm{C}-6,6^{\prime}\right), 61.5\left(\mathrm{C}^{\prime \prime}\right), 20.5\left(\mathrm{COCH}_{3}\right) ;{ }^{1} \mathrm{H}, \delta$ 7.32-7.20 (m, $\left.30 \mathrm{H}, 6 \mathrm{Ph}\right)$, $5.937\left(\mathrm{~m}, 1 \mathrm{H}, \mathrm{OCH}_{2} \mathrm{CH}=\mathrm{CH}_{2}\right), 5.319$ and $5.211\left(2 \mathrm{~m}\right.$, each $\left.1 \mathrm{H}, \mathrm{OCH}_{2} \mathrm{CH}=\mathrm{CH}_{2}\right), 1.988,1.981,1.948$, and 1.924 (4 s, each $3 \mathrm{H}, 4 \mathrm{Ac}$ ).

Allyl 2,3,6-tri-O-benzyl-4-O-[2,3,6-tri-O-benzyl-4-0-(2,3,4-tri-O-benzyl-6-O-trityl- $\beta$-D-glucopyranosyl)- $\beta$-D-glucopyranosyl]- $\beta$-D-glucopyranoside (29). - To a solution of 26 (695 mg, $0.55 \mathrm{mmol}$ ) in $\mathrm{CH}_{2} \mathrm{Cl}_{2}$ $(1 \mathrm{~mL})$ and $\mathrm{MeOH}(5 \mathrm{~mL}$ ) was added sodium methoxide ( $\mathrm{pH} \mathrm{10)}$. After stiring for $2 \mathrm{~h}$, the mixture was neutralised with Dowex-50 $\left(\mathrm{H}^{+}\right)$resin, filtered, and concentrated $(\rightarrow 27)$. To a solution of the residue in pyridine $(50 \mathrm{~mL})$ at $60^{\circ}$ was added trityl chloride $(180 \mathrm{mg}, 0.65 \mathrm{mmol})$, and the mixture was stirred for $20 \mathrm{~h}$ at 600 . Then TLC showed the disappearance of starting material and the formation of a new compound $\left(95: 9 \mathrm{CH}_{2} \mathrm{Cl}_{2}\right.$ $\left.\mathrm{MeOH} ; 28 \boldsymbol{R}_{\mathrm{F}} \mathbf{0 . 3 7}\right) .{ }^{1} \mathrm{H}-\mathrm{NMR}$ data $\left(\mathrm{CDCl}_{3}\right)$ of acetylated $28: \delta 7.45-7.20(\mathrm{~m}, 45 \mathrm{H}, 9 \mathrm{Ph}), 5.937(\mathrm{~m}, 1 \mathrm{H}$, $\left.\mathrm{OCH}_{2} \mathrm{CH}=\mathrm{CH}_{2}\right), 5.319$ and $5.217\left(2 \mathrm{~m}\right.$, each $\left.1 \mathrm{H}, \mathrm{OCH}_{2} \mathrm{CH}=\mathrm{CH}_{2}\right), 1.986,1.979$, and $1.947(3 \mathrm{~s}$, each $3 \mathrm{H}$, $3 \mathrm{Ac})$. After concentration, a solution of the residue in $N, N$-dimethylformamide $(10 \mathrm{~mL})$ was added to a suspension of sodium hydride $(70 \mathrm{mg}, 2.9 \mathrm{mmol})$ in $N, N$-dimethylformamide $(8 \mathrm{~mL})$, and benzyl bromide $(0.25$ $\mathrm{mL}, 2.0 \mathrm{mmol})$ was added dropwise at $0^{\circ}$. After $2 \mathrm{~h}$ TLC (9:1 toluene-acetone) showed the disappearance of the starting compound $\left(R_{\mathrm{F}} 0.29\right)$ and one new compound $\left(R_{\mathrm{F}} 0.40\right)$. Methanol was added, and the solution was diluted with $\mathrm{CH}_{2} \mathrm{Cl}_{2}$ (30 mL), washed with water, aq $10 \%$ sodium hydrogencarbonate, and water, dried $\left(\mathrm{MgSO}_{4}\right)$, filtered, and concentrated. Column chromatography (95:5 toluene-acetone) of the residue gave 29. isolated as a syrup $(175 \mathrm{mg}, 20 \%$ from 26$)$, $[\alpha]_{D}+3^{\circ}\left(c 1, \mathrm{CH}_{2} \mathrm{Cl}_{2}\right) .{ }^{1} \mathrm{H}-\mathrm{NMR}$ data $\left(\mathrm{CDCl}_{3}\right): \delta$ 7.47-7.18 (m, $60 \mathrm{H}, 12 \mathrm{Ph}), 5.949\left(\mathrm{~m}, 1 \mathrm{H}, \mathrm{OCH}_{2} \mathrm{CH}=\mathrm{CH}_{2}\right), 5.324$ and $5.209\left(2 \mathrm{~m}\right.$, each $\left.1 \mathrm{H}, \mathrm{OCH}_{2} \mathrm{CH}=\mathrm{CH}_{2}\right)$.

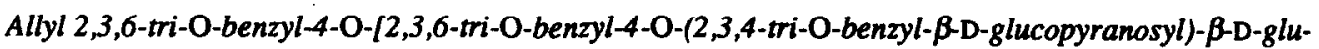
copyranosyl]- $\beta$-D-glucopyranoside (30). - To a solution of $29(175 \mathrm{mg}, 110 \mu \mathrm{mol})$ in $\mathrm{CH}_{2} \mathrm{Cl}_{2}$ (3 mL) were 
added a few drops of aq $70 \%$ perchloric acid, and after stirring for $10 \mathrm{~min}$, solid sodium hydrogencarbonate was added. Then the mixture was diluted with $\mathrm{CH}_{2} \mathrm{Cl}_{2}(3 \mathrm{~mL})$, filtered, washed twice with water, dried $\left(\mathrm{MgSO}_{4}\right)$, filtered, and concentrated. Column chromatography (95:5 toluene-acetone) of the residue afforded 30, isolated as a syrup (119 mg, 80\%), $[\alpha]_{\mathrm{D}}+12^{\circ}\left(c 1, \mathrm{CH}_{2} \mathrm{Cl}_{2}\right), R_{\mathrm{F}} 0.39$ (95:5 toluene-acetone). NMR data $\left(\mathrm{CDCl}_{3}\right):{ }^{13} \mathrm{C}, \delta$ 139.2-138.0 and $128.2-127.0(\mathrm{Ph}), 134.0\left(\mathrm{OCH}_{2} \mathrm{CH}=\mathrm{CH}_{2}\right), 117.0\left(\mathrm{OCH}_{2} \mathrm{CH}=\mathrm{CH}_{2}\right)$, 102.5, 102.4, and 102.2 (C-1,1',1"), 84.5, 82.9, 82.8, 82.6, 81.8, 81.6, 77.8 (2 C), 77.0, 76.4, 76.0, and

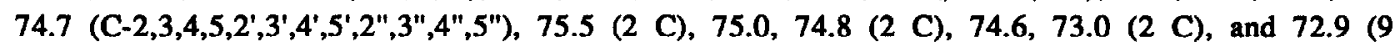
$\left.\mathrm{OCH}_{2} \mathrm{Ph}\right), 70.1\left(\mathrm{OCH}_{2} \mathrm{CH}=\mathrm{CH}_{2}\right), 68.1$ and $67.7\left(\mathrm{C}-6,6^{\prime}\right), 61.7\left(\mathrm{C}-6^{\prime \prime}\right) ;{ }^{1} \mathrm{H}, \delta$ 7.33-7.14 (m, $\left.45 \mathrm{H}, 9 \mathrm{Ph}\right)$, $5.946\left(\mathrm{~m}, 1 \mathrm{H}, \mathrm{OCH}_{2} \mathrm{CH}=\mathrm{CH}_{2}\right), 5.323$ and $5.185\left(2 \mathrm{~m}\right.$, each $\left.1 \mathrm{H}, \mathrm{OCH}_{2} \mathrm{CH}=\mathrm{CH}_{2}\right)$.

Allyl 4-0-[4-O-(2,3,4-tri-O-benzyl- $\beta$-D-glucopyranosyluronic acid)-2,3,6-tri-O-benzyl- $\beta$-D-glucopyranosyl]-2,3,6-tri-O-benzyl- $\beta$-D-glucopyranoside (31). - To a solution of 30 (69 mg, $51 \mu \mathrm{mol}$ ) in acetone ( $3 \mathrm{~mL}$ ) at $0^{\circ}$ was added Jones reagent $(150 \mu \mathrm{L})$. After stirring for $3 \mathrm{~h}$ at $0^{\circ}$, when TLC showed the reaction to be complete (95:5 toluene-acetone; $31 R_{\mathrm{F}} 0.27$ ), the mixture was diluted with water $(5 \mathrm{~mL})$, and the solvent was concentrated for $50 \%$. The solution was extracted with ether $(3 \times 5 \mathrm{~mL})$, and the combined extracts were washed with water, aq $10 \%$ sodium hydrogencarbonate, and water, dried $\left(\mathrm{MgSO}_{4}\right)$, filtered, and concentrated. Column chromatography (95:5 toluene-acetone) of the residue gave 31, isolated as a syrup (53 mg, 75\%), [ $\alpha]_{D}$ $+25^{\circ}\left(\mathrm{c} 1, \mathrm{CH}_{2} \mathrm{Cl}_{2}\right)$. NMR data $\left(\mathrm{CDCl}_{3}\right):{ }^{13} \mathrm{C}, \delta 170.1\left(\mathrm{C}-6^{\prime \prime}\right), 139.2-137.5$ and 128.2-126.9 $(\mathrm{Ph}), 134.0$ $\left(\mathrm{OCH}_{2} \mathrm{CH}=\mathrm{CH}_{2}\right), 117.0\left(\mathrm{OCH}_{2} \mathrm{CH}=\mathrm{CH}_{2}\right), 102.5,102.4$, and $101.6\left(\mathrm{C}-1,1^{\prime}, 1^{\prime \prime}\right), 83.3,82.2,82.6,82.3$, 82.1, 81.5 (3 C), 78.9, 75.8, 74.6, and 73.1 (C-2,3,4,5,2',3',4',5',2",3",4",5"), 75.4, 74.9, 74.8, 74.7, $73.0(2 \mathrm{C})$, and $72.9(3 \mathrm{C})\left(9 \mathrm{OCH}_{2} \mathrm{Ph}\right), 70.1\left(\mathrm{OCH}_{2} \mathrm{CH}=\mathrm{CH}_{2}\right), 68.0$ and $67.6\left(\mathrm{C}-6,6^{\prime}\right) ;{ }^{1} \mathrm{H}, 8$ 7.33-7.14 (m, $45 \mathrm{H}, 9 \mathrm{Ph}), 5.915\left(\mathrm{~m}, 1 \mathrm{H}, \mathrm{OCH}_{2} \mathrm{CH}=\mathrm{CH}_{2}\right), 5.321$ and $5.186\left(2 \mathrm{~m}\right.$, each $\left.1 \mathrm{H}, \mathrm{OCH}_{2} \mathrm{CH}=\mathrm{CH}_{2}\right)$.

4-O-[4-O-( $\beta$-D-glucopyranosyluronic acid)- $\beta$-D-glucopyranosyl]-D-glucopyranose (34). - A mixture of 31 ( $52 \mathrm{mg}, 38 \mu \mathrm{mol})$, palladium (II) chloride $(45 \mathrm{mg}, 254 \mu \mathrm{mol})$ and sodium acetate trihydrate $(36 \mathrm{mg}, 265$ $\mu \mathrm{mol})$ in aq $96 \%$ acetic acid $(10 \mathrm{~mL})$ was sonicated in an ultrasonic cleaner for $72 \mathrm{~h}$. Then TLC $\left(9: 1 \mathrm{CH}_{2} \mathrm{Cl}_{2}\right.$ $\mathrm{MeOH})$ showed the disappearance of starting material $\left(31, R_{\mathrm{F}} 0.44\right)$ and the formation of two new spots $\left(R_{\mathrm{F}}\right.$ 0.40 and 0.29$)$. The mixture was diluted with $\mathrm{CH}_{2} \mathrm{Cl}_{2}(10 \mathrm{~mL})$, filtered, washed with water, aq $10 \%$ sodium hydrogencarbonate, and water, dried $\left(\mathrm{MgSO}_{4}\right)$, filtered, and concentrated. Column chromatography (95:5 $\left.\mathrm{CH}_{2} \mathrm{Cl}_{2}-\mathrm{MeOH}\right)$ of the residue gave $32(19 \mathrm{mg})$ and $33(26 \mathrm{mg}, 51 \%)$, both isolated as a syrup. 33: [ $\left.\alpha\right]_{\mathrm{D}}$ $+40^{\circ}\left(\mathrm{c} 1, \mathrm{CH}_{2} \mathrm{Cl}_{2}\right) .{ }^{13} \mathrm{C}-\mathrm{NMR}$ data $\left(\mathrm{CDCl}_{3}\right): \delta 169.3\left(\mathrm{C}-6{ }^{\prime \prime}\right), 139.3-137.6$ and $129.8-127.0(\mathrm{Ph}), 102.6$ and 101.5 (C-1',1"), 97.2 (C-1ß), 91.2 (C-1 $\alpha$ ), 83.3, 82.5, 82.2, 81.5, 79.9, 78.9 (2 C), 76.7, 75.8, 74.6, 72.9, and $70.2\left(\mathrm{C}-2,3,4,5,2^{\prime}, 3^{\prime}, 4^{\prime}, 5^{\prime}, 2^{\prime \prime}, 3^{\prime \prime}, 4^{\prime \prime}, 5^{\prime \prime}\right), 75.5$ ( $\left.3 \mathrm{C}\right), 75.1,74.8,74.7,73.5,73.2$, and 73.0 (9 $\left.\mathrm{OCH}_{2} \mathrm{Ph}\right), 67.7$ (2 C) (C-6,6').

A solution of $33(17 \mathrm{mg}, 13 \mu \mathrm{mol})$ in 4:1 EtOH-EtOAc ( $8 \mathrm{~mL})$, containing $10 \%$ Pd-C (15 mg), was hydrogenolysed at $1 \mathrm{~kg} / \mathrm{cm}^{2}$ for $8 \mathrm{~h}$, when TLC $\left(1: 1 \mathrm{CH}_{2} \mathrm{Cl}_{2}-\mathrm{MeOH}\right)$ showed the absence of starting material, and the mixture was filtered through Celite, concentrated, and co-concentrated twice with water ( $4 \mathrm{~mL}$ ). The residue was lyophilised to yield 34 , isolated as a white powder $(6 \mathrm{mg}, 90 \%),[\alpha]_{\mathrm{D}}+29^{\circ}\left(c 1, \mathrm{H}_{2} \mathrm{O}\right)$. ${ }^{1} \mathrm{H}-\mathrm{NMR}$ (COSY, HOHAHA) data $\left(\mathrm{D}_{2} \mathrm{O}\right): \delta 5.224(\mathrm{~d}, 0.3 \mathrm{H}, \mathrm{H}-1 \alpha), 4.662$ (d, $\left.0.7 \mathrm{H}, \mathrm{H}-1 \beta\right), 4.548$ (d, $\left.1 \mathrm{H}, \mathrm{H}-1^{\prime}\right)$, 4.538 (d, $\left.1 \mathrm{H}, \mathrm{H}-1^{\prime \prime}\right), 3.972$ (H-6a'), 3.958 (H-6aß), 3.823 (H-6b'), 3.812 (H-6bß), 3.768 (H-5"), 3.581 $(\mathrm{H}-2 \alpha), 3.371\left(\mathrm{H}-2^{\prime}\right), 3.352\left(\mathrm{H}-2^{\prime \prime}\right), 3.283$ (t, $\left.0.7 \mathrm{H}, \mathrm{H}-2 \beta\right) ; J_{1 \alpha, 2} 3.8, J_{1 \beta, 2} 8.0, J_{1^{\prime}, 2^{\prime}} 7.9, J_{1 " 2 "} 8.0 \mathrm{~Hz}$.

Allyl 4-O-l4-O-(2,3-di-O-benzyl-4,6-0-benzylidene- $\beta$-D-glucopyranosyl)-2,3,6-tri-O-benzyl- $\alpha$-D-glucopyranosyl]-2,3,6-tri-O-benzyl- $\beta$-D-galactopyranoside (35).- A mixture of 19 (418 mg, $0.41 \mathrm{mmol}), 10$ (350 $\mathrm{mg}, 0.71 \mathrm{mmol})$, and powdered molecular sieves $(4 \AA, 1.5 \mathrm{~g})$ in dry ether $(20 \mathrm{~mL})$ was cooled to $-70^{\circ}$. After stirring for $20 \mathrm{~min}$, trimethylsilyl trifluoromethanesulfonate $(100 \mu \mathrm{L}, 0.83 \mathrm{mmol})$ was added, and the mixture 
was stirred for $4 \mathrm{~h}$ at $-30^{\circ}$. Then TLC showed the disappearance of 19 and the formation of a new spot $\left(\boldsymbol{R}_{\mathrm{F}}\right.$ $0.28,65: 35$ hexane-EtOAc). The mixture was neutralised with aq $25 \%$ ammonia, diluted with $\mathrm{CH}_{2} \mathrm{Cl}_{2}$ (15 $\mathrm{mL})$, filtered, and concentrated. A solution of the residue in $\mathrm{CH}_{2} \mathrm{Cl}_{2}(10 \mathrm{~mL})$ was washed with water, aq $10 \%$ sodium hydrogencarbonate, and water, dried $\left(\mathrm{MgSO}_{4}\right)$, filtered, and concentrated. Column chromatography (8:2 hexane-EtOAc) of the residue gave $35(302 \mathrm{mg}, 55 \%)$ and $36(84 \mathrm{mg}, 15 \%)$, both isolated as syrups. 35 : $\left.[\alpha]_{D}+75^{\circ}(c), \mathrm{CH}_{2} \mathrm{Cl}_{2}\right)$. NMR data $\left(\mathrm{CDCl}_{3}\right):{ }^{13} \mathrm{C}, \delta 139.3-137.3$ and 128.7-125.8 (Ph), 133.9 $\left(\mathrm{OCH}_{2} \mathrm{CH}=\mathrm{CH}_{2}\right), 117.3\left(\mathrm{OCH}_{2} \mathrm{CH}=\mathrm{CH}_{2}\right), 102.9$ and $102.7\left(\mathrm{C}-1,1^{\prime \prime}\right), 100.8$ and 100.3 (C-1' and $\left.\mathrm{OCHPh}\right)$; ${ }^{1} \mathrm{H}, 8$ 7.37-7.15 (m, $\left.45 \mathrm{H}, 9 \mathrm{Ph}\right), 5.953\left(\mathrm{~m}, 1 \mathrm{H}, \mathrm{OCH}_{2} \mathrm{CH}=\mathrm{CH}_{2}\right), 5.449(\mathrm{~s}, 1 \mathrm{H}, \mathrm{OCHPh}), 5.327$ and 5.184 ( $2 \mathrm{~m}$, each $\left.\left.1 \mathrm{H}, \mathrm{OCH}_{2} \mathrm{CH}=\mathrm{CH}_{2}\right), 5.046\left(\mathrm{~d}, 1 \mathrm{H}, \mathrm{H}-1^{\prime}\right) ; J_{1^{\prime}, 2^{\prime}} 3.5 \mathrm{~Hz} .36:[\alpha]_{\mathrm{D}}+12^{\circ}(c), \mathrm{CH}_{2} \mathrm{Cl}_{2}\right) . \mathrm{NMR}$ data $\left(\mathrm{CDCl}_{3}\right)$ : ${ }^{13} \mathrm{C}, \delta 139.3-137.5$ and $129.0-126.1(\mathrm{Ph}), 134.3\left(\mathrm{OCH}_{2} \mathrm{CH}=\mathrm{CH}_{2}\right), 117.1\left(\mathrm{OCH}_{2} \mathrm{CH}=\mathrm{CH}_{2}\right)$, 103.1, 102.9, and 102.7 (C-1,1',1"), 101.2 (OCHPh); ${ }^{1} \mathrm{H}, \delta$ 7.37-7.20 (m, 45 H, 9 Ph), 5.994 (m, 1 H, $\left.\mathrm{OCH}_{2} \mathrm{CH}=\mathrm{CH}_{2}\right), 5.497(\mathrm{~s}, 1 \mathrm{H}, \mathrm{OCHPh}), 5.320$ and $5.174\left(2 \mathrm{~m}\right.$, each $\left.1 \mathrm{H}, \mathrm{OCH}_{2} \mathrm{CH}=\mathrm{CH}_{2}\right)$.

4-O-(4-O- $\beta$-D-Glucopyranosyl- $\alpha$-D-glucopyranosyl)-D-galactopyranose (38).- To a solution of 35 (97 $\mathrm{mg}, 72 \mu \mathrm{mol})$ in dry $N, N$-dimethylformamide $\left(6 \mathrm{~mL}\right.$ ) at $60^{\circ}$ was added potassium tert-butoxide until the solution turned black. After stirring for $20 \mathrm{~h}$ the solution was diluted with $\mathrm{CH}_{2} \mathrm{Cl}_{2}$ (10 mL), washed twice with water, and concentrated. To a solution of the residue in 9:1 acetone-water $(20 \mathrm{~mL})$ were added mercuric oxide $(12 \mathrm{mg}$ ) and mercuric chloride $(840 \mathrm{mg})$, and the mixture was stirred ovegnight, when TLC (95:5 tolueneacetone) showed the absence of the starting compound, diluted with $\mathrm{CH}_{2} \mathrm{Cl}_{2}(25 \mathrm{~mL})$, and concentrated. A solution of the residue in $\mathrm{CH}_{2} \mathrm{Cl}_{2}(15 \mathrm{~mL})$ was washed with water, aq $10 \%$ potassium iodide, water, aq $10 \%$ sodium hydrogencarbonate, and water, dried $\left(\mathrm{MgSO}_{4}\right)$, filtered, and concentrated. Column chromatography (97:3 toluene-acetone) of the residue afforded 37, isolated as a syrup (67 mg, $71 \%$ ). A solution of 37 (67 mg, $51 \mu \mathrm{mol})$ in 1:1 EtOAc-EtOH $(15 \mathrm{~mL})$, containing $10 \% \mathrm{Pd}-\mathrm{C}(50 \mathrm{mg})$, was hydrogenolysed at $1 \mathrm{~kg} / \mathrm{cm}^{2}$ for 12 $h$, when TLC (4:1 $\left.\mathrm{CH}_{2} \mathrm{Cl}_{2}-\mathrm{MeOH}\right)$ showed the absence of starting material. Then the mixture was filtered through Celite, concentrated, and lyophilised to yield 38, isolated as a white powder $(20 \mathrm{mg}, 80 \%),[\alpha]_{D}$ $+156^{\circ}(c) 1, \mathrm{H}_{2} \mathrm{O}$ ). ${ }^{1} \mathrm{H}-\mathrm{NMR}$ (COSY, HOHAHA) data ( $\left.\mathrm{D}_{2} \mathrm{O}\right): \delta 5.300$ (d, $\left.0.25 \mathrm{H}, \mathrm{H}-1 \alpha\right), 4.935$ and 4.927 (2

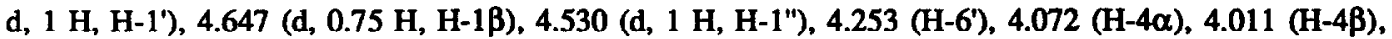

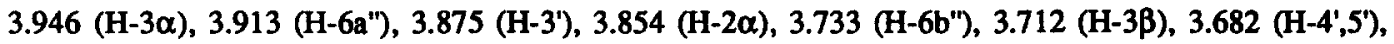
3.587 (H-2'), 3.528 (H-2ß), 3.504 (H-3"), 3.484 (H-5"), $3.416(\mathrm{H}-4 "), 3.319(\mathrm{H}-2 ") ; J_{1 \alpha, 2} 3.9, J_{1 \beta, 2} 7.8$, $J_{1 ', 2} 3.8, J_{1 "}^{\prime \prime 2} 2^{\prime \prime} 8.0 \mathrm{~Hz}$.

Anal. Calc. for $\mathrm{C}_{18} \mathrm{H}_{32} \mathrm{O}_{16} \cdot \mathrm{H}_{2} \mathrm{O}: \mathrm{C}, 41.38 ; \mathrm{H}, 6.56$. Found: $\mathrm{C}, 41.47 ; \mathrm{H}, 6.53$.

4-O-(4-O- $\beta$-D-Glucopyranosyl- $\beta$-D-glucopyranosyl)-D-galactopyranose (40). - To a solution of 36 (48 $\mathrm{mg}, 35 \mu \mathrm{mol}$ ) in dry $N, N$-dimethylformamide $\left(4 \mathrm{~mL}\right.$ ) at $60^{\circ}$ was added potassium tert-butoxide until the solution turned black. After stirring for $20 \mathrm{~h}$ the solution was diluted with $\mathrm{CH}_{2} \mathrm{Cl}_{2}(8 \mathrm{~mL})$, washed twice with water, and concentrated. To a solution of the residue in 9:1 acetone-water $(12 \mathrm{~mL})$ were added mercuric oxide $(5 \mathrm{mg}$ ) and mercuric chloride ( $960 \mathrm{mg}$ ), and the mixture was stirred overnight, when TLC (95:5 toluene-acetone) showed the absence of the starting compound, diluted with $\mathrm{CH}_{2} \mathrm{Cl}_{2}(15 \mathrm{~mL})$, and concentrated. A solution of the residue in $\mathrm{CH}_{2} \mathrm{Cl}_{2}(10 \mathrm{~mL})$ was washed with water, aq $10 \%$ potassium iodide, water, aq $10 \%$ sodium hydrogencarbonate, and water, dried $\left(\mathrm{MgSO}_{4}\right)$, filtered, and concentrated. Column chromatography (97:3 toluene-acetone) of the residue afforded 39 , isolated as a syrup ( $29 \mathrm{mg}, 62 \%)$. A solution of 39 ( $29 \mathrm{mg}$, $22 \mu \mathrm{mol})$ in 1:1 EtOAc-EtOH $(15 \mathrm{~mL})$, containing $10 \%$ Pd-C $(40 \mathrm{mg})$, was hydrogenolysed at $1 \mathrm{~kg} / \mathrm{cm}^{2}$ for 12 $h$, when TLC (4:1 $\left.\mathrm{CH}_{2} \mathrm{Cl}_{2}-\mathrm{MeOH}\right)$ showed the absence of starting material. Then the mixture was filtered through Celite, concentrated, and lyophilised to yield 40 , isolated as a white powder $(8.5 \mathrm{mg}, 77 \%),[\alpha]_{D}$ $+26^{\circ}(c)$ l, $\mathrm{H}_{2} \mathrm{O}$ ). ${ }^{1} \mathrm{H}-\mathrm{NMR}$ data $\left(\mathrm{D}_{2} \mathrm{O}\right)$ : $\delta 5.270$ (d, $\left.0.2 \mathrm{H}, \mathrm{H}-1 \alpha\right), 4.686$ (d, $\left.1 \mathrm{H}, \mathrm{H}-1^{\prime}\right), 4.606$ (d, $0.8 \mathrm{H}, \mathrm{H}-$ 1ß), 4.507 (d, $\left.1 \mathrm{H}, \mathrm{H}-1^{\prime \prime}\right) ; J_{1 \alpha, 2} 3.7, J_{1 ', 2} 7.9, J_{1 \beta, 2} 7.8, J_{1 ", 2 "} 7.9 \mathrm{~Hz}$. 


\section{ACKNOWLEDGMENTS}

This investigation was supported by the Netherlands Foundation for Chemical Research (SON/NWO), the Institute of Molecular Biology and Medical Biotechnology (IMB, Utrecht University), and the Netherlands Innovation Directed Programme for Biotechnology (IOP-b). We like to thank Mr M. J. van Vliet for his help during synthesis, Drs. J. P. M. Lommerse and B. R. Leeflang for recording the 2D ${ }^{1}$ H-NMR spectra, and Drs. J. G. M. van der Ven and A. M. P. van Steijn for recording the ${ }^{13}$ C-NMR spectra.

\section{REFERENCES}

1. Robbins, J. B.; Austrian, R.; Lee, C.-J.; Rastogi, S. C.; Schiffman, G.; Henrichsen, J.; Măkelä, P. H.; Broome, C. V.; Facklam, R. R.; Tiesjema, R. H.; Parke Jr., J. C. J. Infec. Dis. 1983, 148, 1136-1 159.

2. van Dam, J. E. G.; Fleer, A.; Snippe, H. Antonie van Leeuwenhoek 1990, 58, 1-47.

3. Jones, J. K. N.; Perry, M. B. J. Am. Chem. Soc. 1957, 79, 2787-2793.

4. Chernyak, A. Y.; Antonov, K. V. Bioorg. Khim. 1992, 18, 716-725.

5. Takano, T.; Nakatsubo, F.; Murakami, K. Carbohydr. Res. 1990, 203, 341-342.

6. Excoffier, G.; Gagnaire, D.; Utille, J.-P. Carbohydr. Res. 1975, 39, 368-373.

7. Sato, S.; Ito, Y.; Nukada, T.; Nakahara, Y.; Ogawa, T. Carbohydr. Res. 1987, 167, 197-210.

8. Jacquinet, J. C.; Sinaÿ, P. Tetrahedron 1979, 35, 365-371.

9. Garegg, P. J.; Hultberg, H.; Wallin, S. Carbohydr. Res. 1982, 108, 97-101.

10. Gigg, R.; Warren, C. D. J. Chem. Soc. (C) 1968, 1903-1911.

11. Legler, G.; Bause, E. Carbohydr. Res. 1973, 28, 45-52.

12. Ichikawa, Y.; Ichikawa, R.; Kuzuhara, H. Carbohydr. Res. 1985, 141, 273-282.

13. Ichikawa, Y.; Monden, R.; Kuzuhara, H. Carbohydr. Res. 1988, 172, 37-64.

14. Marion, D.; Wüthrich, K. Biochem. Biophys. Res. Commun. 1984, 113, 967-974.

15. Bax, A.; Davis, D. G. J. Magn. Reson. 1985, 65, 355-360.

16. Schmidt, R. R.; Stumpp, M. Liebigs Ann. Chem. 1983, 1249-1256. 